\title{
Acoustic and Vibration Control for an Underwater Structure under Mechanical Excitation
}

\author{
Shi-Jian Zhu, ${ }^{1}$ Xue-Tao Weng, ${ }^{1}$ Hong-Liang Dai, ${ }^{2}$ Yi-Ming Fu, ${ }^{2}$ and Yi-Qi Mao ${ }^{2}$ \\ ${ }^{1}$ Institute of Noise \& Vibration, Naval University of Engineering, Wuhan 430033, China \\ ${ }^{2}$ Department of Engineering Mechanics, College of Mechanical \& Vehicle Engineering, Hunan University, Changsha 410082, China
}

Correspondence should be addressed to Hong-Liang Dai; hldai520@sina.com

Received 28 May 2013; Revised 12 March 2014; Accepted 12 March 2014; Published 30 April 2014

Academic Editor: Hongyi Li

Copyright (C) 2014 Shi-Jian Zhu et al. This is an open access article distributed under the Creative Commons Attribution License, which permits unrestricted use, distribution, and reproduction in any medium, provided the original work is properly cited.

\begin{abstract}
Acoustic and vibration control for an underwater structure under mechanical excitation has been investigated by using negative feedback control algorithm. The underwater structure is modeled with cylindrical shells, conical shells, and circular bulkheads, of which the motion equations are built with the variational approach, respectively. Acoustic property is analyzed by the Helmholtz integration formulation with boundary element method. Based on negative feedback control algorithm, a control loop with a coupling use of piezoelectric sensor and actuator is built, and accordingly some numerical examples are carried out on active control of structural vibration and acoustic response. Effects of geometrical and material parameters on acoustic and vibration properties are investigated and discussed.
\end{abstract}

\section{Introduction}

The reduction of noise emitted by underwater structures has long been a key topic in naval research. This arises from water being a very good sound transmitter, allowing detection of underwater structures by passive sonar over large distances [1]. To absorb the sound energy transmitting through the structure, a coating concerning with sound absorption is always attached to the surface of the structure which shows a typical viscoelastic property. However, discontinuity of material properties between the coatings with substrate always gives rise to interfacial problem as stress concentration and thermal stress discontinuity and consequently results in interfacial damage and delamination. For this purpose, the core consisting of functionally graded material (FGM) is adopted to settle this matter successfully.

Functionally graded materials (FGMs) are microscopically inhomogeneous composite materials, in which the mechanical properties vary smoothly and continuously from one surface to another. The concept of FGM is an alternative to laminated composite materials which show a mismatch in properties at material interfaces. Recently, many researches concerning FGM structures have been made; Rahmani et al.
[2] studied vibration behavior of a sandwich structure with a flexible functionally graded syntactic core. Ávila [3] carried out an experimental investigation of failure of piecewise functionally graded sandwich beams subjected to three-point bending. More researches have studied the properties of FGM structures [4-9]. Here, it needs to indicate, the FGM is introduced to tack with the interfacial problem. But more attention should be paid to the reasonable structure design of whole structure to tackle the problem [10, 11], and this would be our further approach on this problem. The viscoelastic damping was always utilized to affect dynamic response of the structure for some special purposes. By utilizing the material's viscoelastic energy dissipation property, much research has been given on the structural vibration. By controlling the structural vibration, the sound radiated by structural vibration would be attenuated consequently. This method of acoustic control by controlling structural vibration with the use of material's viscoelastic property had been investigated by Ray and Balaji [12], in which an alternative technological solution for passive earthquake control of shear building structures using a viscoelastic material as a core layer under torsion and bending effect had been presented. In the present study, the viscoelastic material is modeled as a linear 
solid-type material to present damping characteristics, and this process of acoustic control is adopted in the acoustic property of an underwater structure.

As for the acoustic control, many methods have been proposed and investigated. An appropriate secondary sound source, which optimally interferes with the primary noise source, was used to affect sound cancellation in earlier active noise control techniques. They commonly used as actuators for noise control applications, for example the traditional loudspeaker. Although this was a highly reliable sound source, it tended to become quite bulky and heavy at low frequencies where active noise control was most effective [13]. For noise control, one means of attempting to reduce the noise was by using passive noise control techniques such as sound and vibration insulation. The high frequency vibration could be repressed by adopting stiffened materials with high damping ratio. But it was considered expensive and unnecessary luxury, especially in military applications. Lin et al. [14] simulated numerically active control of structural acoustic pressure in a rectangular cavity with a flexible beam and the control of structural acoustic pressure and vibration of the beam was implemented by applying the optimal voltage on piezoelectric actuators through an $\mathrm{LQR}$ controller. Considering mass and stiffness of piezoelectric layers and damage effects of composite layers, Fu and Ruan [15] used a negative velocity feedback control algorithm coupling the direct and converse piezoelectric effects to realize the active control and damage detection with a closed control loop. Malgaca [16] investigated vibration control problems using commercial finite element programs and realized integration of control methods into the finite element solutions (ICFES) in ANSYS. The simulation results obtained by the ICFES were compared with the analysis results obtained by the Laplace transform method, and a good agreement was obtained. Lim et al. [17] presented a detailed optimal control design based on the general finite element approach for the integrated design of a structure and its control system. Li et al. $[18,19]$ also have conducted deep research on some control methods, including adaptive control method [18] and output feedback control [19] method, to which the interested readers are referred. However, as far as we know, acoustic and vibration control of an underwater structure under mechanical excitation has not been found in the literature.

In the present analysis, the vibration and acoustic control for an underwater structure is investigated by applying the negative feedback control algorithm. The underwater structure is modeled with cylindrical shells, conical shells, and circular bulkheads, of which the motion equations are built with the variational approach, respectively. A FGM core is considered to tackle the interfacial problem and the analytical equations are established for each part through the variational approach. The acoustic properties are investigated with the Helmholtz integration formulation on surfaces of the underwater structure which has been solved by the boundary element method. A negative feedback control algorithm is adopted to build a control loop to control the vibration and acoustic response of the underwater structure. The numerical examples show that some useful conclusions have been drawn.

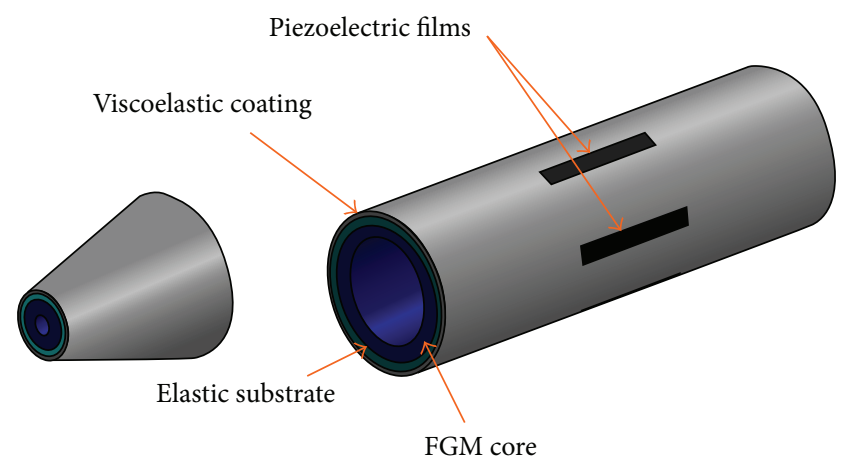

FIGURE 1: Schematic diagram of an underwater structure with piezoelectric films amounted on the hull.

\section{Structural Model of the Underwater Structure}

A geometric model is built according to the reference (Caresta and Kessissoglou [20]) in the present analysis on acoustic and vibration control of an underwater structure. Only half of the underwater structure is modeled as a cylindrical shell with internal bulkhead when considering the symmetry of the underwater structure, the cylindrical shell is closed by a truncated conical shell, which is closed at each end by a circular plate as shown in Figure 1. Three laminated layers, the elastic substrate, FGM core, and viscoelastic coating are considered for the cylindrical and conical shells. Four pairs of piezoelectric films ( $L$ denotes length, $H$ denotes width) are attached to the inner and outer surfaces of the cylindrical shell, which are, respectively, taken as sensors and actuators (as shown in Figure 1), located in the center of the cylindrical shell. The direct and inverse piezoelectric effects are considered for the sensors and actuators. The material properties of the substrate, sandwich core, and coating are considered as isotropic elastic material, FGM, and viscoelastic material, respectively. The surrounding fluid is assumed to be water $\left(\rho_{1}=1000 \mathrm{~kg} / \mathrm{m}^{3}, c_{1}=1480 \mathrm{~m} / \mathrm{s}\right)$ and the filling fluid is considered to be air $\left(\rho_{2}=1.2 \mathrm{Kg} / \mathrm{m}^{3}, c_{1}=\right.$ $344 \mathrm{~m} / \mathrm{s}$ ) at atmospheric pressure and ambient temperature.

\section{Structural Analysis Model of Underwater Structure}

In this part, the constitutive relations are built for elastic, viscoelastic, and piezoelectric materials, respectively. By establishing the equations of displacement energy and kinetic energy of the system and utilizing Euler-Lagrange equation, and basing on the Hamilton theory, the motion equations for the underwater structure are obtained through the variational approach. The general displacement and strain fields are, respectively, given firstly in Section 3.1 and then in Section 3.2; here, different types of material properties are built for different layers of the underwater structure. In Section 3.3, motion equations for the whole structure are obtained with the variational approach. 
3.1. Displacement Field and Strain Filed. The geometrical nonlinearity in the elastic deformation framework is considered, and three parts of the structure (cylindrical shell, conical shell, and circular bulkhead) have the same general displacement-strain forms. According to the classical nonlinear elastic theory, the strain tensor of any point in the elastic structure can be denoted as

$$
\varepsilon_{i j}=\frac{1}{2}\left(u_{i, j}+u_{j, i}+u_{k, i} u_{k, i}\right), \quad(i, j=1,2,3),
$$

where $\varepsilon_{i j}$ denotes the Green strain component, $u_{i}$ is the displacement component, and $u_{i, j}$ indicates the derivative of displacement components with respect to coordinate. With no complementary indication, the Cartesian coordinate is referred to for the definition of the variables components.

\subsection{Constitutive Relations of the Underwater Structure}

3.2.1. Constitutive Relation for the Elastic Material. The isotropic property of the material is considered in the present analysis; therefore, for a linear elastic medium, the general constitutive relation is written as

$$
\sigma_{i j}=E_{i j k l} \varepsilon_{k l}
$$

where $\sigma_{i j}$ denotes the stress component and $E_{i j k l}$ denotes the stiffness coefficient. Considering the plane problem, three independent material parameters are considered for isotropic materials, while five independent material parameters are considered for anisotropic materials.

3.2.2. Constitutive Relation for FGM Core. To tackle the interfacial problem, a FGM core is considered between the elastic substrate and coating which are both taken as the same materials. The FGM core's material contains elastic properties varying along the thickness according to volume fraction law, and the FGM can be expressed as follows:

$$
p_{f}=(1-V(z)) p_{m}+V(z) p_{c}
$$

where $p_{f}$ is the effective material property of the FGM and $p_{m}$ and $p_{c}$ denote properties of the metal and sound-absorbing materials, respectively. $V(z)$ is the volume fraction of metal constituent of FGM. The volume fraction is assumed to follow a power law function as

$$
V(z)=\left(\frac{z-R-h_{s}}{h_{f}}\right)^{n} .
$$

Then the elastic constitutive relationship for the FGM can be given as

$$
\sigma_{i j}=E_{i j k l}(z) \varepsilon_{k l},
$$

where $E_{i j k l}(z)$ denotes the elastic modulus varying along the thickness. From (5), it can be found that the FGM constitutive relation is related to the $z$ coordinate which differs from the homogeneous material shown in (2).
3.2.3. Constitutive Relation for the Viscoelastic Material. The viscoelastic property of coating is considered as what contributes to dissipation of energy due to its transverse shear deformation, and the energy dissipation will cause the vibration attenuation of the underwater structure and consequently control acoustic response radiated by vibration of the underwater structure. The viscoelastic constitutive is given in the following.

Before the introduction of constitutive relation of the viscoelastic material, the following convolution integration is defined as

$$
\varphi \otimes \frac{d \psi}{d t}=\varphi(t) \psi(0)+\int_{0}^{t} \varphi(t-\tau) \frac{d \psi(\tau)}{d \tau} d \tau .
$$

The following two constitutive relations for the viscoelastic materials are always adopted in the viscoelastic analysis:

$$
\varepsilon(t)=F(t) \otimes \frac{d \sigma}{d t} \quad \text { or } \quad \sigma(t)=E(t) \otimes \frac{d \varepsilon}{d t},
$$

where $E(t)$ denotes the stress relaxation modulus and $F(t)$ denotes the creep compliance, with $E F=1$ for the elastic material.

The following constitutive relation is mainly adopted in the present analysis; it is written as

$$
\sigma_{i j}(t)=\int_{-\infty}^{t} E_{i j k l}(t-\tau) \frac{d \varepsilon_{k l}(\tau)}{d \tau} d \tau .
$$

Here, as for the stress relaxation modulus $E_{i j k l}(t)$, there exists the symmetry of $i$ and $j, k$, and $l$. Therefore, there are 36 independent components of relaxation modulus $E_{i j k l}(t)$. If an isotropic material is considered, there are only two independent stress relaxation functions, and $E_{i j k l}$ is a fourorder isotropic tensor, and

$$
E_{i j k l}(t)=\lambda(t) \delta_{i j} \delta_{k l}+\mu(t) \delta_{i k} \delta_{j l}+\nu(t) \delta_{i l} \delta_{j k} .
$$

Then (8) can be rewritten in the simplified form as

$$
\begin{aligned}
\sigma_{i j}(t)= & \delta_{i j} \int_{-\infty}^{t} \lambda(t-\tau) \frac{d \varepsilon_{k k}(\tau)}{d \tau} \\
& +2 \int_{-\infty}^{t} \mu(t-\tau) \frac{d \varepsilon_{i j}(\tau)}{d \tau} d \tau,
\end{aligned}
$$

where $\lambda(t)$ and $\mu(t)$ denote stress relaxation functions.

For simplicity, the standard linear solid-type material is considered, where the relaxation function is written in terms of the Prony series as

$$
E(t-\tau)=E_{\infty}+\sum_{m} E_{m} e^{-\alpha_{m}\left(t-t_{1}\right)},
$$

where $E_{\mathrm{\infty}}$ is the long-time Young modulus of the viscoelastic material and $1 / \alpha_{m}$ denotes the relaxation time.

3.2.4. Constitutive Relation for the Piezoelectric Material. The active control of the radiated acoustic response can be realized by controlling the vibration of the structure as 
done by Sharma et al. [21] with use of piezoelectric sensors and actuators. The constitutive relation for the piezoelectric material is given as

$$
\begin{gathered}
\sigma_{i j}=c_{i j k l} \varepsilon_{k l}+e_{i j k} E_{k} \\
D_{i}=e_{i k l} \varepsilon_{k l}+k_{i j} E_{j},
\end{gathered}
$$

where $c_{i j k l}, e_{i k l}$, and $k_{i j}$ are, respectively, the elastic, piezoelectric, and dielectric permittivity constants and $D_{i}$ denotes electric displacement.

The electric field-potential relation is denoted as

$$
E_{i}=-\phi_{, i}
$$

where $\phi$ denotes the electric potential.

3.3. Motion Equations for the Underwater Structure. The motion equations of the underwater structure are obtained with the energy variational approach in this section. The strain-displacement relations are set differently for three parts (cylindrical shell, conical shell, and circular bulkhead), so the energy functions and the motion equilibrium relations are established, respectively. The variational process has been omitted and only the final results are given in order to keep the integrity and clearness of the paper structure. In this section, the energy function of the system is built firstly for three parts, respectively, and the variation approaches are carried out for the motion equations based on the Hamilton theory.

3.3.1. The Energy Function and Motion Equations for the Cylindrical Shell. At first, the total systematic energy can be given for the cylindrical shell according to the displacementstrain relation and the constitutive relation for different materials. As for the cylindrical shell as shown in Figure 2, the following strain-displacement relation is adopted as

$$
\varepsilon_{x}=\varepsilon_{x}^{0}+z \kappa_{x}^{0}, \quad \varepsilon_{y}=\varepsilon_{y}^{0}+z \kappa_{y}^{0}, \quad \varepsilon_{x y}=\varepsilon_{x y}^{0}+z \kappa_{x y}^{0},
$$

where

$$
\begin{gathered}
\varepsilon_{x}^{0}=u_{, x}^{0}+\frac{1}{2}\left(w_{, x}^{0}\right)^{2}, \quad \varepsilon_{y}^{0}=v_{, y}^{0}-\frac{w^{0}}{R}+\frac{1}{2}\left(w_{, y}^{0}\right)^{2}, \\
\varepsilon_{x y}^{0}=u_{, y}^{0}+v_{, x}^{0}+w_{, x}^{0} w_{, y}^{0}, \quad \kappa_{x}^{0}=-w_{, x x}^{0}, \\
\kappa_{y}^{0}=-w_{, y y}^{0}, \quad \kappa_{x y}^{0}=-2 w_{, x y}^{0} .
\end{gathered}
$$
follows:

Then, the total kinematical energy can be written as

$$
\begin{aligned}
T= & T^{(s)}+T^{(f)}+T^{(c)}+T^{(p)} \\
= & \iiint_{\Omega^{(s)}} \frac{1}{2} \rho^{(s)} \dot{u}_{i}^{2} d \Omega+\iiint_{\Omega^{(f)}} \frac{1}{2} \rho^{(f)} \dot{u}_{i}^{2} d \Omega \\
& +\iiint_{\Omega^{(c)}} \frac{1}{2} \rho^{(c)} \dot{u}_{i}^{2} d \Omega+\iiint_{\Omega^{(p)}} \frac{1}{2} \rho^{(p)} \dot{u}_{i}^{2} d \Omega,
\end{aligned}
$$

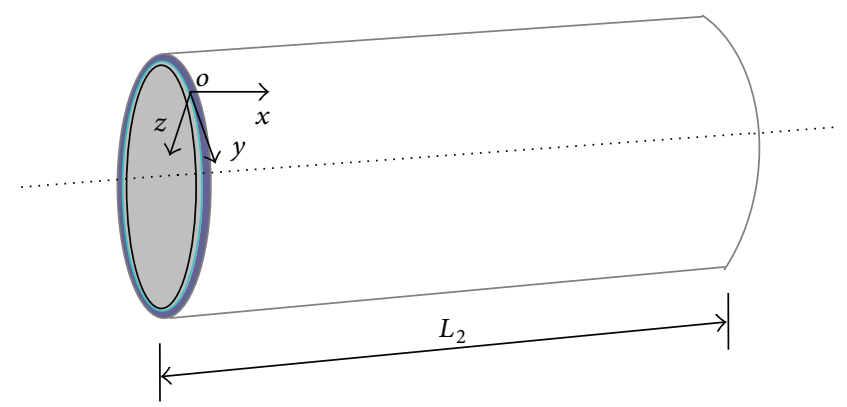

FIGURE 2: Configuration of the cylindrical shell.

where superscripts $s, f, c, p$ indicate, respectively, the substrate, FGM core, viscoelastic coating, and piezoelectric films, and the same denotations are adopted in the following process. The total kinematical energy is the summation of the kinematical energy for different layers, and the same form is adopted in the following for the displacement energy and strain energy.

The total displacement energy for the external force of the cylindrical shell can be given as

$$
V=V^{(s)}+V^{(f)}+V^{(c)}+V^{(p)} .
$$

The displacement energy for different layers can be written as

$$
\begin{aligned}
V^{(s)} & =-\iiint_{\Omega^{(s)}} \frac{1}{2} B_{i}^{(s)} u_{i} d \Omega-\iint_{S(s)}{ }^{(s)} T_{i}^{(v)} u_{i} d S \\
V^{(f)} & =-\iiint_{\Omega^{(f)}} \frac{1}{2} B_{i}^{(f)} u_{i} d \Omega-\iint_{S(f)}{ }^{(f)} T_{i}^{(v)} u_{i} d S \\
V^{(c)} & =-\iiint_{\Omega^{(c)}} \frac{1}{2} B_{i}^{(c)} u_{i} d \Omega-\iint_{S(c)}{ }^{(c)} T_{i}^{(v)} u_{i} d S \\
V^{(p)} & =-\iiint_{\Omega^{(p)}} \frac{1}{2} B_{i}^{(p)} u_{i} d \Omega-\iint_{S(p)}{ }^{(p)} T_{i}^{(v)} u_{i} d S,
\end{aligned}
$$

where $T_{i}^{(\nu)}$ denotes the surface force component and $B_{i}$ denotes the body force component. $\Omega$ and $S$ indicate, respectively, the integration volume and area.

The total strain energy can be given as

$$
\begin{aligned}
U= & U^{(s)}+U^{(f)}+U^{(c)}+U^{(p)} \\
= & \iiint_{\Omega^{(s)}} \frac{1}{2} \tau_{i j}^{(s)} \varepsilon_{i j}^{(s)} d \Omega+\iiint_{\Omega^{(f)}} \frac{1}{2} \tau_{i j}^{(f)} \varepsilon_{i j}^{(f)} d \Omega \\
& +\iiint_{\Omega^{(c)}} \frac{1}{2} \tau_{i j}^{(c)} \varepsilon_{i j}^{(c)} d \Omega \\
& +\iiint_{\Omega^{(p)}} \frac{1}{2}\left(\tau_{i j}^{(p)} \varepsilon_{i j}^{(p)}-k_{i j} E_{i} E_{j}\right) d \Omega,
\end{aligned}
$$

where $\tau_{i j}$ is the stress component and $\varepsilon_{i j}$ is the strain component, $e_{k i j}$ is the dielectric parameter, and $E_{k}$ is the electric field component.

As for the hull of the underwater structure, the cylindrical shell is subjected to external mechanical load along the 
Shock and Vibration

5

normal direction and the only polarization along thickness of the piezoelectric layer is considered in the present analysis. So only the surface forces along the normal direction applied on the viscoelastic coating are considered. What is more, the acoustic pressure acting on the coating surface is considered as the external force which will change the total displacement energy of the system.

When the laminated structure is considered as shown in Figure 2, only the surface force applied on coating surface and the piezoelectric load on the piezoelectric films are considered. Then neglecting the body force effect, the total external force displacement energy for the cylindrical shell can be written in the following form:

$$
\begin{aligned}
V= & V^{(c)}+V^{(p)}=-\iint_{S^{(c)}} p w d S-\iint_{S^{(c)}} p^{\text {acous }} w d S \\
& -q w-\iint_{S^{(p)}} Q \phi d S,
\end{aligned}
$$

where $p$ is the external mechanical pressure on the surface of the viscoelastic coating, $q$ is the concentrated mechanical force acting on the whole system, $Q$ is the applied electric charge, $\phi$ is the polarization electrical potential, and $p^{\text {acous }}$ is the acoustic pressure acting on the piezoelectric film.

For the cylinder shell, the Lagrange function can be given as

$$
\text { Lair }=K+V+U
$$

Basing on the Hamilton theory, the following energy function is defined within the time span $\left[t_{1}, t_{2}\right]$ :

$$
I=\int_{t_{1}}^{t_{2}}(K+V+U) d t=0 .
$$

The Hamilton theory requires

$$
\begin{aligned}
\delta^{(1)} I & =\delta^{(1)} \int_{t_{1}}^{t_{2}}(K+V+U) d t \\
& =\int_{t_{1}}^{t_{2}}\left(\delta^{(1)} K+\delta^{(1)} V+\delta^{(1)} U\right) d t .
\end{aligned}
$$

Substituting (18), (19), and (20) into (21) and utilizing (14), the energy variational forms in (23) can be given as follows:

$$
\begin{aligned}
\delta^{(1)} V= & -\iint_{S^{(c)}} p \delta w d S-\iint_{S^{(c)}} p^{\text {acous }} \delta w d S-q \delta w \\
& -\iint_{S^{(p)}} Q \delta \phi d S \\
\delta^{(1)} T= & -\iint_{S} \frac{1}{2}\left(\rho^{(s)} h^{(s)}+\rho^{(f)} h^{(f)}+\rho^{(c)} h^{(c)}\right) \\
& \times\left(u_{, t t} \delta u+v_{, t t} \delta v+w_{, t t} \delta w\right) d S
\end{aligned}
$$

$$
\begin{aligned}
& \delta^{(1)} U=\frac{1}{2} \iint_{S}\left(\int_{h_{b}^{(s)}}^{h_{t}^{(s)}} E_{11}^{(s)}+\int_{h_{b}^{(f)}}^{h_{t}^{(f)}} E_{11}^{(f)}\right) \\
& \times\left(u_{, x}+\frac{1}{2}\left(w_{, x}\right)^{2}-z w_{, x x}\right)^{2} d h \\
& +\left(\int_{h_{b}^{(s)}}^{h_{t}^{(s)}} E_{12}^{(s)}+\int_{h_{b}^{(f)}}^{h_{t}^{(f)}} E_{12}^{(f)}\right) \\
& \times\left(u_{, x}+\frac{1}{2}\left(w_{, x}\right)^{2}-z w_{, x x}\right) \\
& \times\left(v_{, y}-\frac{w}{R}+\frac{1}{2}\left(w_{, y}\right)^{2}-z w_{, y y}\right) d h \\
& +\left(\int_{h_{b}^{(c)}}^{h_{t}^{(c)}} e_{13} E_{3}\right) \\
& \times\left(u_{, x}+\frac{1}{2}\left(w_{, x}\right)^{2}-z w_{, x x}\right) d h \\
& +\left(\int_{h_{b}^{(s)}}^{h_{t}^{(s)}} E_{12}^{(s)}+\int_{h_{b}^{(f)}}^{h_{t}^{(f)}} E_{12}^{(f)}\right) \\
& \times\left(u_{, x}+\frac{1}{2}\left(w_{, x}\right)^{2}-z w_{, x x}\right) \\
& \times\left(v_{, y}-\frac{w}{R}+\frac{1}{2}\left(w_{, y}\right)^{2}-z w_{, y y}\right) d h \\
& +\left(\int_{h_{b}^{(s)}}^{h_{t}^{(s)}} E_{22}^{(s)}+\int_{h_{b}^{(f)}}^{h_{t}^{(f)}} E_{22}^{(f)}\right) \\
& \times\left(v_{, y}-\frac{w}{R}+\frac{1}{2}\left(w_{, y}\right)^{2}-z w_{, y y}\right)^{2} d h \\
& +\left(\int_{h_{b}^{(c)}}^{h_{t}^{(c)}} e_{23} E_{3}\right) d h \\
& \times\left(v_{, y}-\frac{w}{R}+\frac{1}{2}\left(w_{, y}\right)^{2}-z w_{, y y}\right) \\
& +\left(\int_{h_{b}^{(s)}}^{h_{t}^{(s)}} E_{66}^{(s)}+\int_{h_{b}^{(f)}}^{h_{t}^{(f)}} E_{66}^{(f)}\right) \\
& \times\left(u_{, y}+v_{, x}+w_{, x} w_{, y}-2 w_{, x y}\right)^{2} d h \\
& +\frac{1}{2} \int_{h_{b}^{(s)}}^{h_{t}^{(s)}}\left(k_{11}^{(s)} E_{1}^{2}+k_{22}^{(s)} E_{2}^{2}+k_{33}^{(s)} E_{3}^{2}\right) d h \\
& +\left(\int_{h_{b}^{(c)}}^{h_{t}^{(c)}} E_{11}^{(c)}\right) \otimes\left(u_{, x}+\frac{1}{2}\left(w_{, x}\right)^{2}-z w_{, x y}\right) \\
& \times\left(u_{, x}+\frac{1}{2}\left(w_{, x}\right)^{2}-z w_{, x x}\right) d h \\
& +\left(\int_{h_{b}^{(c)}}^{h_{t}^{(c)}} E_{12}^{(c)}\right) \otimes\left(u_{, x}+\frac{1}{2}\left(w_{, x}\right)^{2}-z w_{, x x}\right)
\end{aligned}
$$




$$
\begin{aligned}
& \times\left(v_{, y}-\frac{w}{R}+\frac{1}{2}\left(w_{, y}\right)^{2}-z w_{, y y}\right) d h \\
& +\left(\int_{h_{b}^{(c)}}^{h_{t}^{(c)}} E_{13}^{(c)} E_{3}\right) \otimes\left(u_{, x}+\frac{1}{2}\left(w_{, x}\right)^{2}-z w_{, x x}\right) d h \\
& +\left(\int_{h_{b}^{(c)}}^{h_{t}^{(c)}} E_{12}^{(c)}\right) \otimes\left(u_{, x}+\frac{1}{2}\left(w_{, x}\right)^{2}-z w_{, x x}\right) \\
& \times\left(v_{, y}-\frac{w}{R}+\frac{1}{2}\left(w_{, y}\right)^{2}-z w_{, y y}\right) d h \\
& +\left(\int_{h_{b}^{(c)}}^{h_{t}^{(c)}} E_{22}^{(c)}\right) \otimes\left(v_{, y}-\frac{w}{R}+\frac{1}{2}\left(w_{, y}\right)^{2}-z w_{, y y}\right) d h \\
& +\left(\int_{h_{b}^{(c)}}^{h_{t}^{(c)}} e_{23} E_{3}\right) \otimes\left(v_{, y}-\frac{w}{R}+\frac{1}{2}\left(w_{, y}\right)^{2}-z w_{, y y}\right) d h \\
& +\left(\int_{h_{b}^{(c)}}^{h_{t}^{(c)}} E_{66}^{(c)}\right) \otimes\left(u_{, y}+v_{, x}+w_{, x} w_{, y}-2 w_{, x y}\right) d h d S,
\end{aligned}
$$

where $h_{b}^{(i)}$ and $h_{t}^{(i)}$ indicate the $z$-coordinate of the bottom and top surfaces of the $i$ layer.

3.3.2. The Energy Function and Motion Equations for the Conical Shell and Circular Bulkhead. The analogous deducing process is adopted following the cylindrical shell as for the energy function and motion equation of a conical shell and circular bulkhead (as shown in Figures 3 and 4). The energy functions are built firstly for the conical shell and circular bulkhead.

In the present analysis, only one layer of the elastic circular bulkhead is considered. As for the conical shell, threelayer laminated structure is considered, and no piezoelectric control effect is applied on the structural vibration of the conical shell. The energy variation for the conical shell and circular bulkhead can be given as follows.

The total displacement energy of the circular bulkhead under external mechanical and acoustic pressure can be given as

$$
\begin{aligned}
\Pi^{(\text {bulkhead })}= & \iiint_{\Omega} \frac{1}{2} \tau_{i j} \varepsilon_{i j} d \Omega-\iiint_{\Omega} \frac{1}{2} B_{i}^{(c)} \dot{u}_{i} d \Omega \\
& -\iint_{S}{ }^{(c)} T_{i}^{\left({ }^{(v)}\right.} \dot{u}_{i} d S .
\end{aligned}
$$

The kinematical energy for the circular bulkhead can be given as

$$
T^{(c)}=\iiint_{\Omega} \rho \dot{u}_{i}^{2} d \Omega .
$$

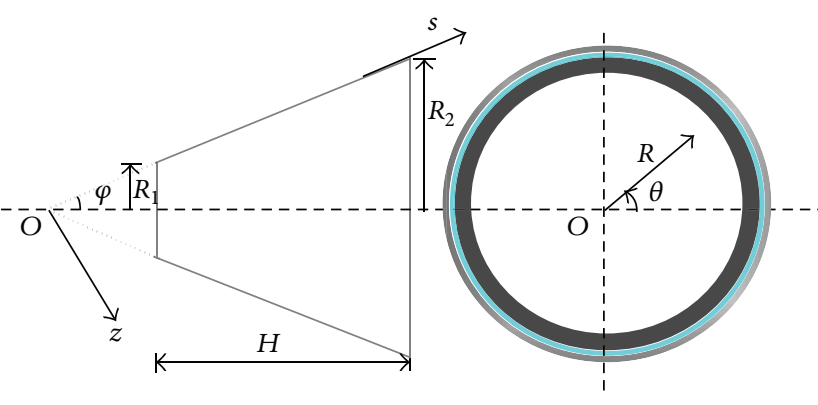

Viscoelastic coating

FGM core

Elastic substrate

FIGURE 3: Configuration of the conical shell and the cross-section.

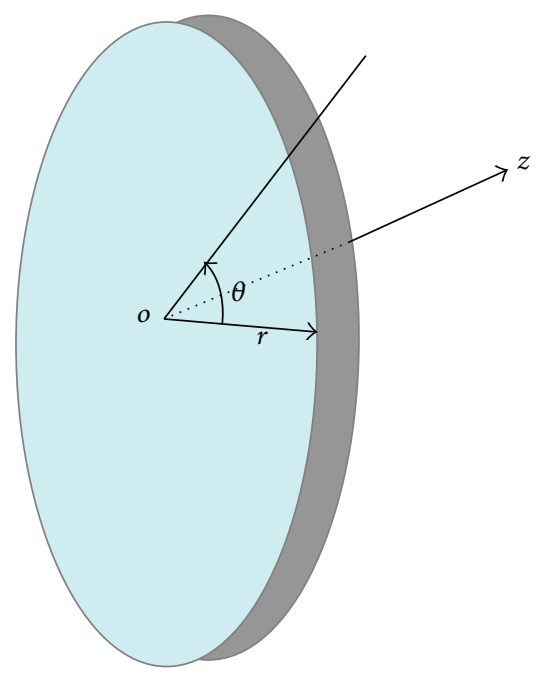

FIGURE 4: Configuration of the circular bulkhead.

Then, the Hamilton theory written in the following form can be obtained

$$
\begin{gathered}
\delta^{(c)} \int_{t_{1}}^{t_{2}}\left(\iiint_{\Omega} \frac{1}{2} \tau_{i j} \varepsilon_{i j} d \Omega-\iiint_{\Omega} \frac{1}{2} B_{i}^{(c)} \dot{u}_{i} d \Omega-\iint_{S}{ }^{(c)} T^{(v)} \dot{u}_{i} d S\right. \\
\left.+\iiint_{\Omega} \frac{1}{2} \rho \dot{u}_{i}^{2} d \Omega\right) d t=0 .
\end{gathered}
$$

The following displacement-strain relations for the circular bulkhead with the Cartesian coordinate are expressed as (as shown in Figure 4)

$$
\begin{aligned}
\varepsilon_{r} & =u_{, r}+\frac{1}{2}\left(w_{, r}\right)^{2}-z w_{, r r} \\
\varepsilon_{\theta} & =\frac{1}{r}\left(u+v_{, \theta}\right)+\frac{1}{2 r^{2}}\left(w_{, \theta}\right)^{2}-\frac{z}{r}\left(w_{, r}+w_{, \theta \theta}\right) \\
\varepsilon_{r \theta} & =\frac{1}{r}\left(u_{, \theta}-v\right)+v_{, r}+\frac{1}{r} w_{, r} w_{, \theta}-z\left(\frac{1}{r}\left(w_{, r \theta}-w_{, \theta}\right)+w_{, \theta r}\right),
\end{aligned}
$$


where $\varepsilon_{r}, \varepsilon_{\theta}$, and $\varepsilon_{r \theta}$ are strain components of any points of the circular bulkhead and $u, v$, and $w$ are displacement components.

Considering the acoustic and mechanical pressure acting on the surface of the conical shell, the total displacement energy for the system under piezoelectric control can be given as

$$
\begin{aligned}
\Pi^{(\text {conical })}= & \iiint_{\Omega} \frac{1}{2} \tau_{i j} \varepsilon_{i j} d \Omega-\iiint_{\Omega} \frac{1}{2} k_{i j} E_{i} E_{j} d \Omega \\
& -\iiint_{\Omega} \frac{1}{2} B_{i}^{(c)} \dot{u}_{i} d \Omega-\iint_{S}{ }^{(c)} T_{i}^{(\nu)} \dot{u}_{i} d S .
\end{aligned}
$$
as

The kinematical energy for the conical shell can be given

$$
T^{(c)}=\iiint_{\Omega} \rho \dot{u}_{i}^{2} d \Omega .
$$

Then similar to the previous process of dealing with the energy function of the conical shell and the circular bulkhead, the Hamilton theory written in the following form can be obtained:

$$
\begin{aligned}
\delta^{(1)} \int_{t_{1}}^{t_{2}}\left(\iiint_{\Omega} \frac{1}{2} \tau_{i j} \varepsilon_{i j} d \Omega-\iiint_{\Omega} \frac{1}{2} k_{i j} E_{i} E_{j} d \Omega\right. \\
\quad-\iiint_{\Omega} \frac{1}{2} B_{i}^{(c)} \dot{u}_{i} d \Omega \\
\left.\quad-\iint_{S}{ }^{(c)} T^{(v)} \dot{u}_{i}+\iiint_{\Omega} \frac{1}{2} \rho \dot{u}_{i}^{2} d \Omega\right) d S d t=0 .
\end{aligned}
$$

The following displacement-strain relations for the conical shell are built as follows:

$$
\begin{aligned}
\varepsilon_{s}= & u_{, s}+\frac{1}{2}\left(w_{, s}\right)^{2}+w_{, s} \bar{w}_{, s}-z w_{, s s} \\
\varepsilon_{\theta}= & \frac{1}{s}\left(u+v_{, \theta} \csc \phi-w \cot \phi\right)+\frac{1}{s^{2}}\left[\frac{1}{2}\left(w_{, \theta}\right)^{2}+w_{, \theta} \bar{w}_{, \theta}\right] \csc ^{2} \phi \\
& -z\left(\frac{1}{s^{2}} w_{, \theta \theta} \csc ^{2} \phi+\frac{1}{s} w_{, s}\right) \\
\varepsilon_{s \theta}= & \frac{1}{s} u_{, \theta} \csc \phi+v_{, s}-\frac{v}{s}+\frac{1}{s}\left(w_{, \theta} \bar{w}_{, s}+w_{, s} \bar{w}_{, \theta}+w_{, s} w_{, \theta}\right) \csc \phi \\
& -z\left(\frac{2}{s^{2}} w_{, \theta}-\frac{1}{s} w_{, s \theta}\right) \csc \phi .
\end{aligned}
$$

After the variation processes are applied on (28) and (31), the motion equilibrium equations can be obtained for the cylindrical shell, conical shell, and circular bulkhead, respectively, in terms of displacement function as well as the boundary conditions.

\subsubsection{Displacement Function and Boundary/Connection Con-} ditions. The solutions to the motion equation built separately for the cylindrical shell, conical shell, and circular bulkhead are obtained with the Galerkin method.
The displacement functions for the conical shell are set as follows:

$$
\begin{aligned}
& u^{c}=u^{c}\left(x^{c}\right) \cos \left(n \theta^{c}\right)=\sum_{n=0}^{\infty} U_{n}^{c} \cdot X_{n}^{c} \cos \left(n \theta^{c}\right) \\
& v^{c}=v^{c}\left(x^{c}\right) \cos \left(n \theta^{c}\right)=\sum_{n=0}^{\infty} V_{n}^{c} \cdot X_{n}^{c} \cos \left(n \theta^{c}\right) \\
& \phi^{c}=\phi^{c}\left(x^{c}\right) \cos \left(n \theta^{c}\right)=\sum_{n=0}^{\infty} \Phi_{n}^{c} \cdot X_{n}^{c} \cos \left(n \theta^{c}\right) \\
& w^{c}=w^{c}\left(x^{c}\right) \cos \left(n \theta^{c}\right)=\sum_{n=0}^{\infty} W_{n}^{c} \cdot X_{n}^{c} \cos \left(n \theta^{c}\right),
\end{aligned}
$$

where the vectors $U_{n}^{c}, V_{n}^{c}, \Phi_{n}^{c}, W_{n}^{c}$ are

$$
\begin{aligned}
& U_{n}^{c}=\left[u_{1}^{c}\left(x^{c}\right), u_{2}^{c}\left(x^{c}\right), \ldots, u_{8}^{c}\left(x^{c}\right)\right] \\
& V_{n}^{c}=\left[v_{1}^{c}\left(x^{c}\right), v_{2}^{c}\left(x^{c}\right), \ldots, v_{8}^{c}\left(x^{c}\right)\right] \\
& \Phi_{n}^{c}=\left[\phi_{1}^{c}\left(x^{c}\right), \phi_{2}^{c}\left(x^{c}\right), \ldots, \phi_{8}^{c}\left(x^{c}\right)\right] \\
& W_{n}^{c}=\left[w_{1}^{c}\left(x^{c}\right), w_{2}^{c}\left(x^{c}\right), \ldots, w_{8}^{c}\left(x^{c}\right)\right], \\
& X_{n}^{c}=\left[\begin{array}{llllllll}
a_{0} & a_{1} & b_{0} & b_{1} & c_{0} & c_{1} & c_{2} & c_{3}
\end{array}\right]^{T} .
\end{aligned}
$$

The displacement functions for the cylindrical shell are set as follows:

$$
\begin{aligned}
& u^{s}=\sum_{n=0}^{\infty} \sum_{i=1}^{8} C_{n, i}^{s} W_{n, i}^{s} e^{j k_{n, i} x} \cos (n \theta) \\
& v^{s}=\sum_{n=0}^{\infty} \sum_{i=1}^{8} G_{n, i}^{s} W_{n, i}^{s} e^{j k_{n, i} x} \sin (n \theta) \\
& \phi^{s}=\sum_{n=0}^{\infty} \sum_{i=1}^{8} \Phi_{n, i}^{s} W_{n, i}^{s} e^{j k_{n, i} x} \cos (n \theta) \\
& w^{s}=\sum_{n=0}^{\infty} \sum_{i=1}^{8} W_{n, i}^{s} i^{j k_{n, i} x} \cos (n \theta),
\end{aligned}
$$

where $C_{n, i}^{s}=U_{n, i}^{s} / W_{n, i}^{s}, G_{n, i}^{s}=V_{n, i}^{s} / W_{n, i}^{s}$, and $\Phi_{n, i}^{s}=\phi_{n, i}^{s} / W_{n, i}^{s}$.

The displacement functions for the circular bulkhead are set as follows:

$$
\begin{aligned}
w^{p} & =\sum_{n=0}^{\infty}\left(A_{n, 1}^{p} J_{n}\left(k_{B}^{p} r\right)+A_{n, 2} I_{n}\left(k_{B}^{p} r\right)\right) \cos \left(n \theta^{p}\right) \\
u^{p} & =\sum_{n=0}^{\infty}\left(B_{n, 1}^{p} \frac{\partial J_{n}\left(k_{L}^{p} r\right)}{\partial r}+\frac{n B_{n, 2} J_{n}\left(k_{B}^{p} r\right)}{r}\right) \cos \left(n \theta^{p}\right) \\
v^{p} & =-\sum_{n=0}^{\infty}\left(\frac{n B_{n, 1}^{p} J_{n}\left(k_{L}^{p} r\right)}{r}+B_{n, 2} \frac{\partial J_{n}\left(k_{B}^{p} r\right)}{\partial r}\right) \sin \left(n \theta^{p}\right),
\end{aligned}
$$

where $J_{n}$ and $I_{n}$, respectively, are the Bessel function and the modified Bessel function of the first kind, and the bending 
wave number and wave numbers for in-plane waves can be written as

$$
\begin{gathered}
k_{B}^{p}=\left(\frac{\rho^{p} \omega^{2} h^{p}}{D^{p}}\right)^{1 / 4}, \quad k_{L}^{p}=\omega\left[\frac{\rho^{p}\left(1-v_{p}^{2}\right)}{E^{p}}\right]^{1 / 2}, \\
k_{T}^{p}=\omega\left[\frac{2 \rho_{p}\left(1+v_{p}\right)}{E^{p}}\right]^{1 / 2} .
\end{gathered}
$$

By using (33), (35), and (36), the motion equations for the circular bulkhead, conical shell, and cylindrical shell can be obtained in terms of the displacement function. The whole problem is iteratively solved in time span, and the geometrical nonlinear items are dealt with by the second extrapolation method as follows:

$$
(x \cdot y)_{J}=(x)_{J} \cdot(y)_{J_{p}},
$$

where $(y)_{J_{p}}$ is the average value of those obtained in previous two iterations. For the initial iterative step, it can be determined by using the quadratic extrapolation; that is,

$$
(y)_{J_{p}}=A(y)_{J-1}+B(y)_{J-2}+C(y)_{J-3}
$$

and for different iterative steps, the coefficients $A, B$, and $C$ can be expressed as follows:

$$
\begin{array}{lll}
J=1: A=1, & B=0, & C=0 \\
J=2: A=2, & B=-1, & C=0 \\
J=3: A=3, & B=-3, & C=1 .
\end{array}
$$

The iteration item in the equations can be written as follows by using the Newmark method. The time is divided into small time step $\tau$, and for the $J$ th step, the following equations can be got:

$$
\begin{aligned}
\left(W_{, \tau \tau}\right)_{J} & =\frac{4\left(W_{J}-W_{J-1}\right)}{(\Delta \tau)^{2}}-\frac{4\left(W_{, \tau}\right)_{J-1}}{\Delta \tau}-\left(W_{, \tau \tau}\right)_{J-1} \\
\left(W_{, \tau}\right)_{J} & =\left(W_{, \tau}\right)_{J-1}+\frac{1}{2}\left[\left(W_{, \tau \tau}\right)_{J}+\left(W_{, \tau \tau}\right)_{J}\right](\Delta \tau) .
\end{aligned}
$$

After the dealing process by the above methods, the following motion equations can be obtained for the underwater structure for any time step:

$$
\begin{gathered}
{[M]\{\ddot{X}\}+[K]^{(e)}\{X\}+[K]^{(e \phi)}\{\Phi\}+[K]^{(v)} \otimes X_{m n}=\{F\}} \\
{[K]^{(e \phi)}\{X\}+[K]^{(\phi \phi)}\{\Phi\}=\{Q\} .}
\end{gathered}
$$

Here $[M]$ is the mass matrix, $[K]^{e}$ is the stiffness matrix concerning layers of substrate, FGM core, and piezoelectric films, and $[K]^{e \phi}$ is the stiffness matrix concerning the viscoelastic coating. $\{F\}$ which are the external forces consist of piezoelectric load, mechanical load, and acoustic pressure. $\{X\}$ are generalized displacement components, and

$$
\begin{aligned}
X_{m n}=[ & U_{m n}^{(\mathrm{cyl})}, V_{m n}^{(\mathrm{cyl})}, W_{m n}^{(\mathrm{cyl})}, \Phi_{m n}^{(\mathrm{cyl})}, U_{m n}^{(\mathrm{cir})}, V_{m n}^{(\mathrm{cir})}, \\
& \left.W_{m n}^{(\mathrm{cir})}, \Phi_{m n}^{(\mathrm{cir})}, U_{m n}^{(\mathrm{con})}, V_{m n}^{(\mathrm{con})}, W_{m n}^{(\mathrm{con})}, \Phi_{m n}^{(\mathrm{con})}\right] .
\end{aligned}
$$

This equation can be resolved with the Galerkin method, and the dynamic response for the whole system can be obtained.

For generality, the $e_{\tau}$ represents the stiffness coefficient $k_{m n}^{(\nu)}, f$ refers to the unknown displacement variables $X$, and the viscoelastic coating is assumed as standard linear solid in the present model. Dividing time $\tau$ equally into small time segments $d \tau$ and utilizing the Newton-Cotes trapezoidal formula, the following relations can be obtained:

$$
\begin{aligned}
e \otimes f_{i, j}= & e(0) f_{i, j}(t)+\int_{0}^{t} \dot{e}(t-\tau) f_{i, j}(\tau) d \tau \\
= & e(0) f_{i, j}(t)+\int_{0}^{t}-\alpha_{1} e_{1} e^{-\alpha_{1} t} e^{\alpha_{1} t} f_{i, j}(\tau) d \tau \\
= & e(0) f_{i, j}(t)+\left(-\alpha_{1} e_{1} e^{-\alpha_{1} t}\right) \\
& \times\left[\int_{0}^{\Delta t}+\int_{\Delta t}^{2 \Delta t}+\cdots+\int_{(N-1) \Delta t}^{N \Delta t}\right] \\
= & e(0) f_{i, j}(t)+\left(-\alpha_{1} e_{1} e^{-\alpha_{1} t}\right) \frac{\Delta t}{2} \\
& \times\left[\left(e^{\alpha_{1} \Delta t} f_{i, j}(\Delta t)+e(0) f_{i, j}(0)+e^{2 \alpha_{1} \Delta t} f_{i, j}(2 \Delta t)\right.\right. \\
& \quad+e^{\alpha_{1} \Delta t} f_{i, j}(\Delta t)+\cdots+e^{N \alpha_{1} \Delta t} f_{i, j}(N \Delta t) \\
& \left.\quad+e^{(N-1) \alpha_{1} \Delta t} f_{i, j}((N-1) \Delta t)\right]
\end{aligned}
$$

It needs to be stated here, and the material constant is given as

$$
E^{(v)}=E_{p}+E_{L} e^{-\alpha \tau t},
$$

where $E^{(v)}$ is the elastic modulus of the viscoelastic coating varying with time, $E_{p}$ and $E_{L}$ are the material constants, and $\alpha$ is the viscoelastic constant.

3.4. Boundary and Continuity Conditions. Considering the change of curvature between the cylindrical shell and conical shell, the following notation was introduced:

$$
\begin{aligned}
& \bar{u}_{c}=u^{c} \cos \alpha-w^{c} \sin \alpha \\
& \bar{w}_{c}=w^{c} \cos \alpha-w^{c} \sin \alpha .
\end{aligned}
$$


TABLE 1: Geometrical sizes of the underwater structure.

\begin{tabular}{lc}
\hline Parameters & Value \\
\hline Hull radius $\left(R_{2}\right)$ & $3.25 \mathrm{~m}$ \\
Hull length $\left(L_{2}\right)$ & $45.0 \mathrm{~m}$ \\
Coating thickness $\left(h_{c}\right)$ & $0.025 \mathrm{~m}$ \\
FGM core thickness $\left(h_{f}\right)$ & $0.015 \mathrm{~m}$ \\
Substrate thickness $\left(h_{s}\right)$ & $0.05 \mathrm{~m}$ \\
Conical shell length $\left(L_{1}\right)$ & $5.0 \mathrm{~m}$ \\
Radius of the conical shell $\left(R_{1}\right)$ & $1.0 \mathrm{~m}$ \\
Length of piezoelectric film $(L)$ & $2.0 \mathrm{~m}$ \\
Width of piezoelectric film $(H)$ & $0.4 \mathrm{~m}$ \\
\hline
\end{tabular}

TABLE 2: Material parameters for the metal substrate.

\begin{tabular}{lcc}
\hline & Parameters & Metal substrate \\
\hline Elastic modulus & $E(\mathrm{GPa})$ & 210 \\
Shear modulus & $G_{12}(\mathrm{GPa})$ & 115 \\
Shear modulus & $G_{23}, G_{13}(\mathrm{GPa})$ & 89.6 \\
Density & $\rho\left(\mathrm{Kg} / \mathrm{m}^{3}\right)$ & 7800 \\
Poisson' ratio & $\mu$ & 0.3 \\
\hline
\end{tabular}

TABLE 3: Material parameters for the piezoelectric material (PZT4).

\begin{tabular}{lcc}
\hline & \multicolumn{2}{c}{ Piezoelectric (PZT4) } \\
\hline & $C_{11}$ & $132 \times 10^{9}$ \\
& $C_{12}$ & $71 \times 10^{9}$ \\
Material constants $\left(\mathrm{N} / \mathrm{m}^{2}\right)$ & $C_{33}$ & $115 \times 10^{9}$ \\
& $C_{13}$ & $73 \times 10^{9}$ \\
& $C_{55}$ & $73 \times 10^{9}$ \\
\hline Poisson's ratio & \multicolumn{2}{c}{0.35} \\
\hline Mass density $\left(\mathrm{Kg} / \mathrm{m}^{3}\right)$ & & $7.5 \times 10^{3}$ \\
\hline & $e_{31}$ & -4.1 \\
Piezoelectric constants $\left(\mathrm{C} / \mathrm{m}^{2}\right)$ & $e_{32}$ & -4.1 \\
& $e_{33}$ & 14.1 \\
& $e_{15}$ & 10.5 \\
\hline \multirow{2}{*}{ Dielectric constants $(\mathrm{F} / \mathrm{m})$} & $k_{11}$ & $7.124 \times 10^{-9}$ \\
& $k_{22}$ & $7.124 \times 10^{-9}$ \\
& $k_{33}$ & $5.841 \times 10^{-9}$ \\
\hline
\end{tabular}

Here $u_{c}$ and $w_{c}$ are the displacement components of the conical shell in the connecting place and the boundary conditions and continuous conditions are given as

$$
\begin{gathered}
u^{s}=\bar{u}_{c}=w^{p}, \quad w^{s}=\bar{w}_{c}=u^{p}, \quad v^{s}=v^{c}=v^{p}, \\
-N^{p}-N_{x}^{c} \cos \alpha+w_{x}^{p}=0 \\
-\left(N_{x}^{s}+\frac{M_{x}^{s}}{a}\right)+\left(N_{x}^{c}+\frac{M_{x}^{c}}{a}\right)+N_{x}^{p}=0 \\
-M_{x}^{s}-M_{x}^{c}-M_{x}^{p}=0
\end{gathered}
$$

where the superscripts $s, c$, and $p$ indicate that the corresponding variables are concerning the cylindrical shell, conical shell, and circular bulkhead, respectively.

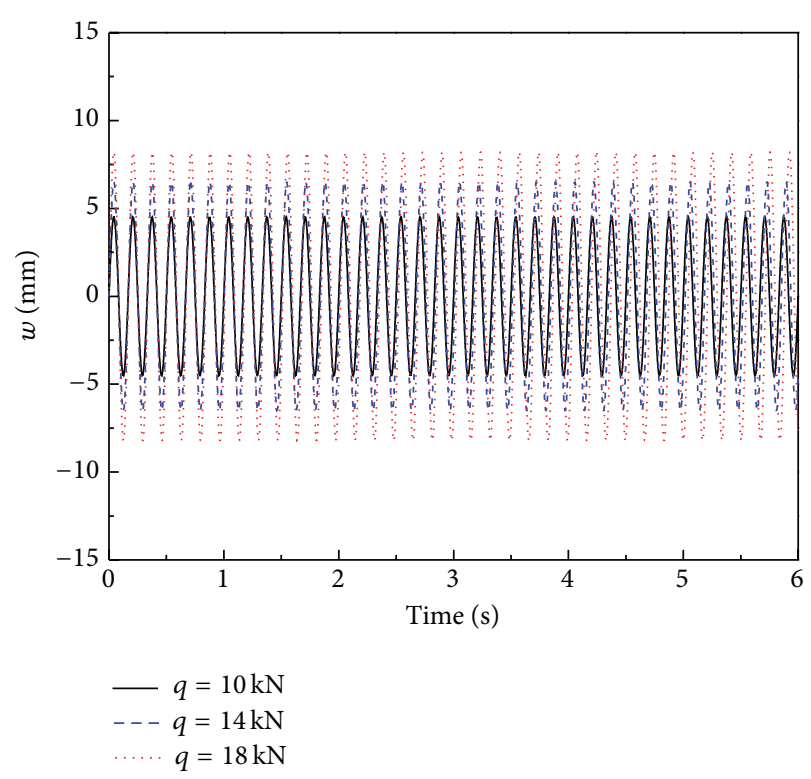

FIGURE 5: Dynamic response of the underwater structure when different loads are applied.

\section{Dynamic Response of the Underwater Structure under Mechanical Excitation}

Dynamic response of the underwater structure under mechanical excitation has been firstly given. The geometrical and material parameters are, respectively, taken as Tables 1 and 2. The piezoelectric parameters are given in Table 3 and the viscoelastic material parameters are listed as follows:

$$
\begin{array}{r}
E_{1}^{(\nu)}=\left(9.75+0.25 e^{-\alpha_{1} t}\right) \times 210 \mathrm{GPa}, \quad \alpha_{1}=0.022, \\
E_{2}^{(\nu)}=\left(0.32+0.68 e^{-\alpha_{2} t}\right) \times 210 \mathrm{GPa}, \quad \alpha_{2}=0.024, \\
G_{12}^{(v)}=\left(0.07+0.16 e^{-\alpha_{12} t}\right) \times 210 \mathrm{GPa}, \quad \alpha_{12}=0.026 \\
\nu=0.3, \rho=7800 \mathrm{~kg} / \mathrm{m}^{3} .
\end{array}
$$

Figure 5 shows dynamic response of the underwater structure under harmonic mechanical force $p=1.0 \times$ $\sin (2 \pi \omega t)(\mathrm{kN})$. It can be found from the calculated results that the maximum amplitude of vibration for the underwater structure happens at the center of the cylindrical shell, and the displacement-time curves reveal that the vibration frequency is a little smaller than that of the excitation frequency, which can be ascribed to the viscoelastic property of the coating. As shown in Figure 6 with a thicker coating, this phenomenon is more obvious. To get a full comprehension of the effect of the viscoelastic properties on dynamic characteristic of the underwater structure, a more explicable comparison has been carried out when considering the coating material to be the linear elastic material $(\alpha=0)$. The force is kept for a period on the surface of the underwater structure and then taken away. Afterwards, the underwater structure undergoes free vibration, and this vibration will theoretically continue for an infinite time when no damping effect is 


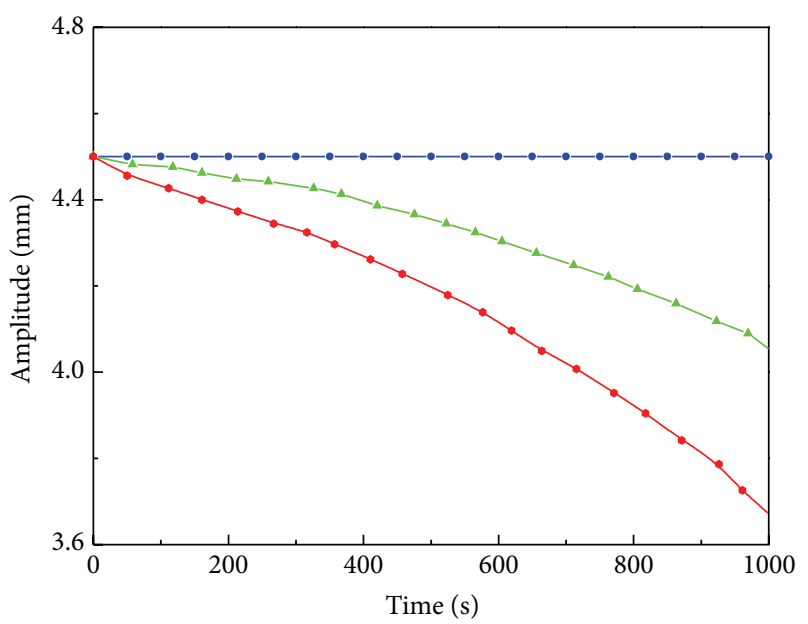

$$
\begin{aligned}
& \alpha=5 \\
& -\bullet-h^{(c)}=10 \\
& -\Delta-h^{(c)}=20 \\
& -\bullet-h^{(c)}=30
\end{aligned}
$$

FIGURE 6: Effect of thickness of the coating on vibration amplitude of the structure.

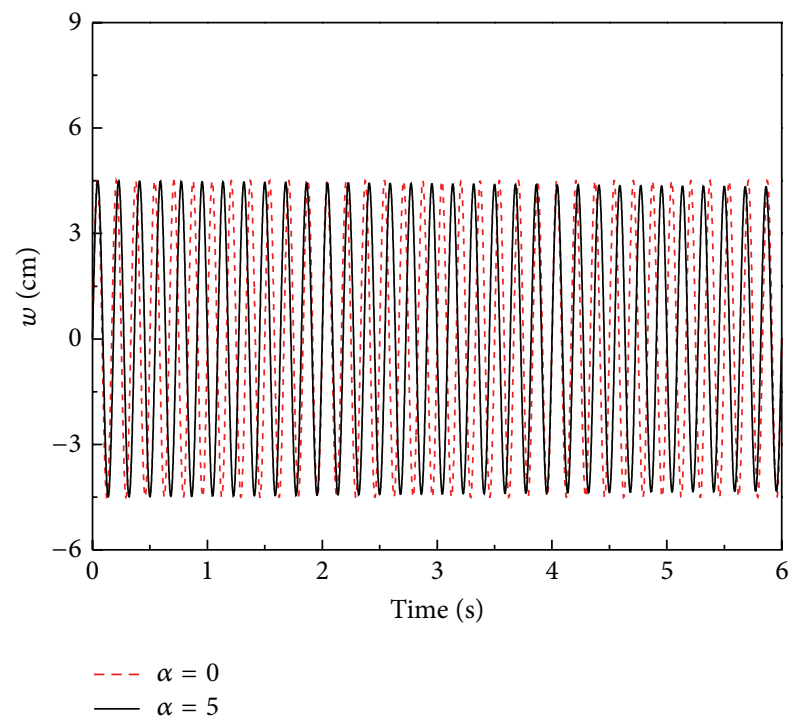

FIGURE 7: Dynamic response of the underwater structure with different viscoelastic material parameters.

considered. However, as far as we know, viscoelastic property of the material exhibits a damping effect on the underwater structure's vibration. From the comparison results of dynamic response of the underwater structure at the center of the cylindrical shell, the displacement of the cylindrical shell is a little small and the frequency also decreases, and it could be due to the energy dissipation of the coating's viscoelastic property. This phenomenon can be proved by the results in Figures 7 and 8. In these two figures, it can be seen that the vibration amplitude decreases as the time prolongs and it will be fully attenuated with the time longer enough.

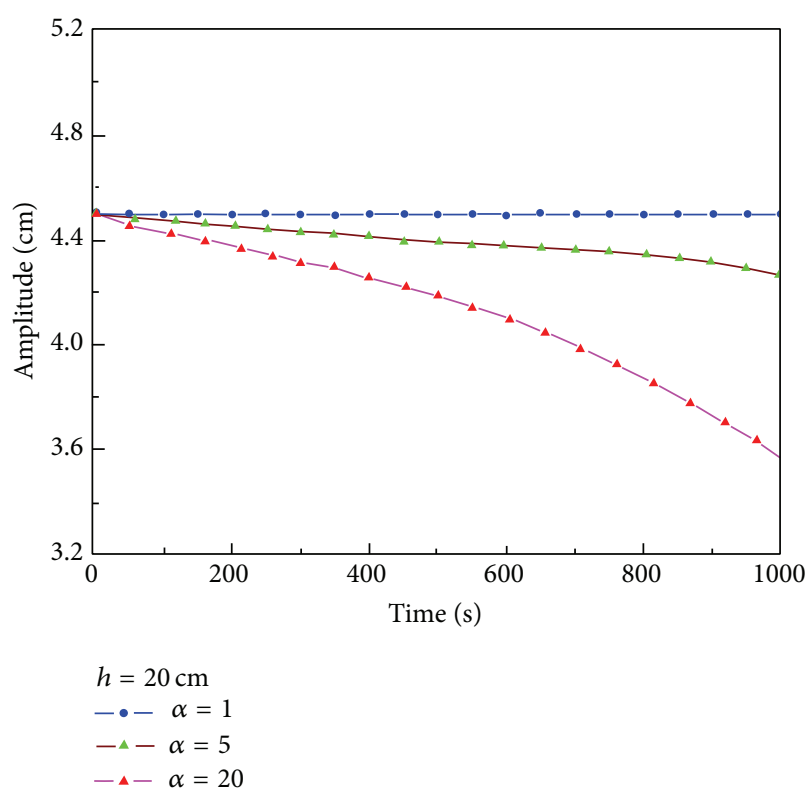

FIGURE 8: Viscoelastic damping effect on vibration amplitude of the underwater structure with different material parameters.

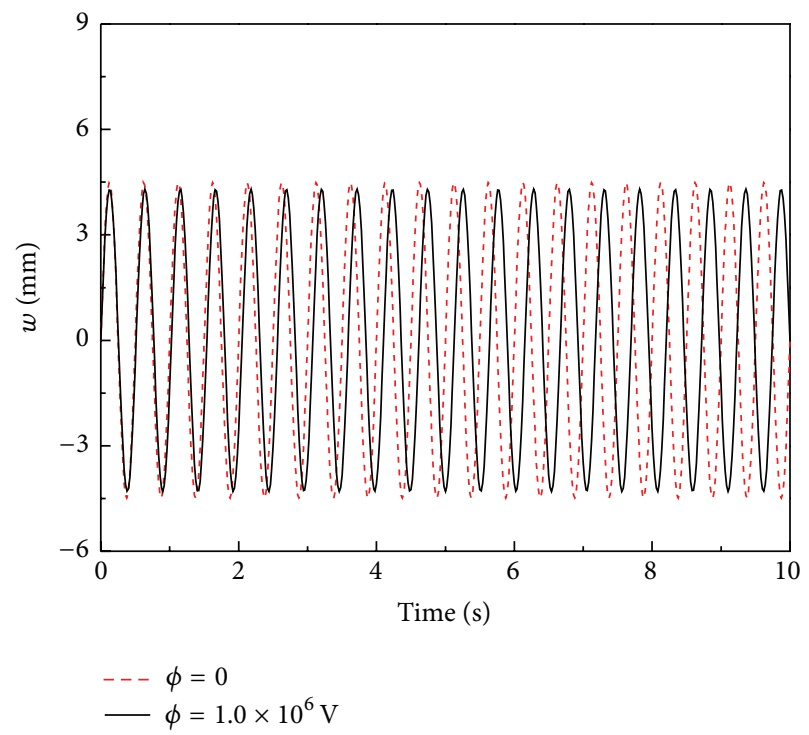

FIGURE 9: Applied negative piezoelectric effect on dynamic response of the underwater structure.

The dynamic response of the underwater structure has been investigated when applying the external piezoelectric potential. From (12), it can be seen that the electric field is generated by the piezoelectric load along the thickness of piezoelectric films, and the change of the resultant force for the underwater structure is caused by the electric field.

Figures 9, 10, and 11 show the effect of the external potential on the dynamic response of the underwater structure. It can be seen from the figures that the positive piezoelectric potential causes the increasing of the vibration amplitude, the 


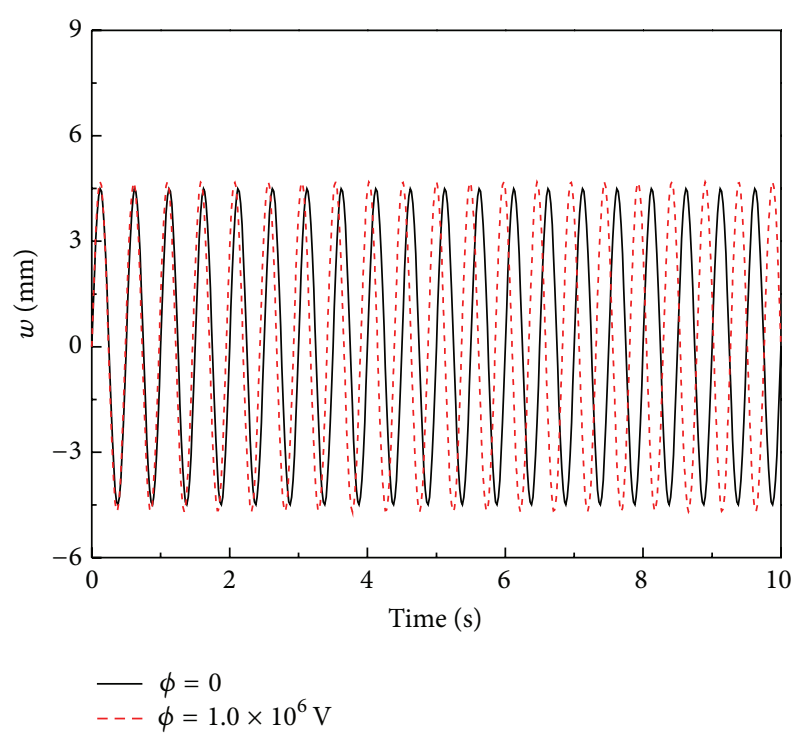

FIGURE 10: Applied negative piezoelectric effect on dynamic response of the underwater structure.

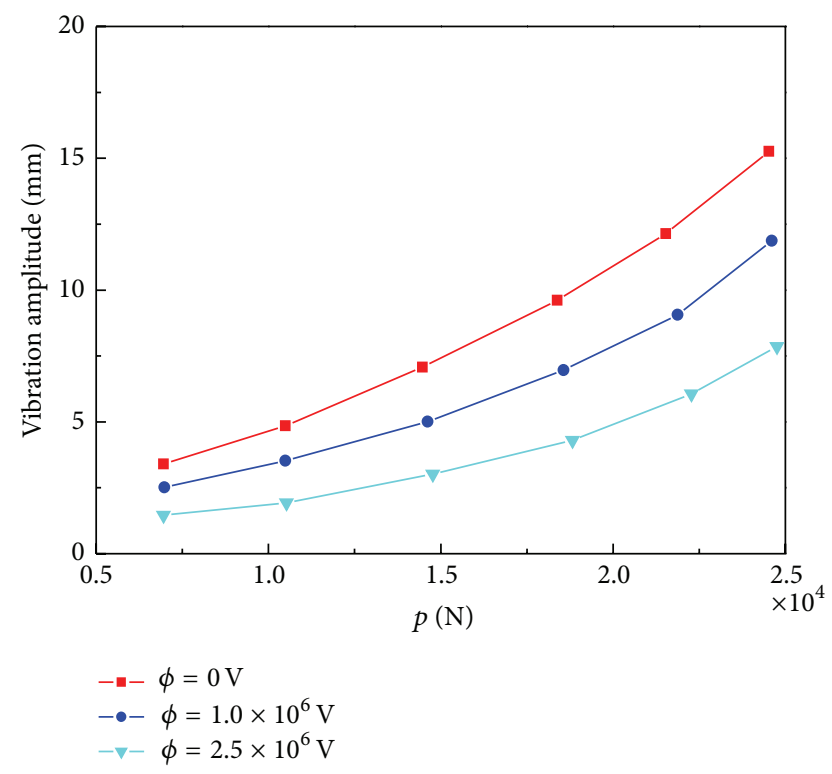

FIGURE 11: Effect of piezoelectric potential on the vibration amplitude of the underwater structure.

negative electric potential causes the decreasing of the vibration amplitude, and it is feasible to attenuate the vibration of the underwater structure by applying a suitable piezoelectric potential.

\section{Acoustic Response of the Underwater Structure under Mechanical Excitation}

In this section, the Helmholtz integral formulation is used to investigate the radiating acoustic properties of the underwater structure under mechanical excitation.
5.1. Helmholtz Integral Formulation. It is assumed that the shell is closed at both ends with circular bulkheads, and an acoustic baffle covers outside the circular bulkhead. The wave equation for the acoustic pressure known as the Helmholtz equation is given by Zhang and $\mathrm{Li}$ [22]:

$$
\nabla^{2} p(r)+k^{2} p(r)=-q(r),
$$

where $p(r)$ is the acoustic pressure; $q(r)$ is the acoustic source in the domain; $k$ is the wave number $\omega / c$; here $\omega$ is the frequency and $c$ is the speed of sound, as denoted in Zhang and $\mathrm{Li}$ [22], the Green's function for the three-dimensional acoustic medium should satisfy the following equation:

$$
\nabla^{2} G\left(r \mid r_{s}\right)+k^{2} G\left(r \mid r_{s}\right)=-\delta\left(r-r_{s}\right) .
$$

The solution to the equation and its normal derivative are given by

$$
\begin{gathered}
G\left(r \mid r_{s}\right)=\frac{e^{-i k R}}{4 \pi R} \\
\frac{\partial G\left(r \mid r_{s}\right)}{\partial n}=-\left[\frac{\cos \theta}{4 \pi R^{2}}+\frac{i k \cos \theta}{4 \pi R}\right] e^{-i k R},
\end{gathered}
$$

where $r_{s}$ and $r$ are the acoustic source location and the observation point, respectively. $R$ is the distance between $r$ and $r_{s} . \theta$ is the angle between the normal direction $n$ and the vector joining $r$ and $r_{s}$.

Some research is carried out by using the divergence theorems [23], and the following boundary integration equations can be obtained:

$$
\begin{aligned}
C(r) & p(r) \\
= & \frac{1}{4 \pi} \int_{s_{0}}\left(P\left(r_{s}\right) \frac{\partial G\left(r \mid r_{s}\right)}{\partial n_{s}}-G\left(r \mid r_{s}\right) \frac{\partial p\left(r_{s}\right)}{\partial n_{s}}\right) d s_{0} \\
& +\int_{\Omega} b\left(r_{s}\right) G\left(r \mid r_{s}\right) d \Omega,
\end{aligned}
$$

where $C(r)$ takes values of $1,0.5$ or 0 depends on whether the observation point $r$ is within the acoustic medium, on the surface $s_{0}$, or outside the acoustic medium, respectively. Here the surface $s_{0}$ encloses the acoustic medium in the three-dimensional space $\Omega$ of the underwater structure. Adopting the same assumption as Zhang and Li [22] that the acoustic energy radiated by the shell is finite, therefore, the acoustic pressure and its gradient vanish as $r \rightarrow \infty$. Under this assumption, the surface integration needs only to be computed on the outer boundary of the underwater structure.

The boundary element method is applied to calculate the boundary integral equations. Two-type six-node quadrilateral element is built as shown in Figure 5. The linear interpolating function along $x$ direction and quadratic interpolating function along $x$ direction are considered. Let us divide the 
surface $S$ into a collection of $M$ boundary elements $S_{i}$, and (53) can be rewritten as

$$
\begin{aligned}
\sum_{i=1}^{M} & {\left[\int \frac{\partial G\left(r_{j} \mid r_{s}\right)}{\partial n_{s}} N\left(r_{s}\right) d S\right] p_{i} } \\
& \quad-\sum_{i=1}^{M}\left[\int_{s_{i}} G\left(r_{j} \mid r_{s}\right) N\left(r_{s}\right) d S\right] q_{i} \\
= & -C_{j} p\left(r_{j}\right)+p_{q}\left(r_{j}\right),
\end{aligned}
$$

where $i$ indicates the source element, $j$ indicates the receiver node, and $N\left(r_{s}\right)$ is the shape function. The nodal variable vectors $p_{i}$ and $q_{i}$ of the $i$ th element are defined as

$$
p_{i}=\left\{\begin{array}{c}
p\left(r_{i, 1}\right) \\
p\left(r_{i, 2}\right) \\
\vdots \\
p\left(r_{i, k}\right)
\end{array}\right\}, \quad q_{i}=\left\{\begin{array}{c}
\frac{\partial p\left(r_{i, 1}\right)}{\partial n_{s}} \\
\frac{\partial p\left(r_{i, 2}\right)}{\partial n_{s}} \\
\vdots \\
\frac{\partial p\left(r_{i, k}\right)}{\partial n_{s}}
\end{array}\right\},
$$

where $r_{i, k}$ is the $k$ th node of the $i$ th element and $k$ is the number of nodes of the element.

Then (54) can be written as

$$
p_{q}\left(r_{j}\right)-C_{j} p\left(r_{j}\right)=\sum_{i=1}^{M} B_{i j} p_{i}+\sum_{i=1}^{M} \mathrm{E}_{i j} q_{i}
$$

and here the element matrices are

$$
\begin{aligned}
B_{i j} & =\int_{s_{i}} \frac{\partial G\left(r_{j} \mid r_{s}\right)}{\partial n_{s}} N\left(r_{s}\right) d S, \\
E_{i j} & =\int_{s_{i}} G\left(r_{j} \mid r_{s}\right) N\left(r_{s}\right) d S .
\end{aligned}
$$

Attention needs to be paid here; the shape function of the boundary element for the cylindrical shell differs from that for the conical shell though the boundary dealing process (49)-(57) is written in the same form.

The acoustic boundary of the underwater structure can be set as

$$
\begin{aligned}
& q=0, \quad \text { the surface without motion } \\
& q=\rho \omega^{2} w_{n}, \quad \text { the surface with vibration, }
\end{aligned}
$$

where $\rho$ is the mass density and $w_{n}$ is the normal displacement of the surface vibrating at the frequency $\omega$.

By coupling with the boundary element method and Galerkin method, the acoustic pressure can be calculated on the surface and in the far field. With the given motion equation in forms of displacement functions of the underwater structure, the acoustic response of the underwater structure can be obtained with the boundary element method when the acoustic vibration is coupled on the surfaces by the relations

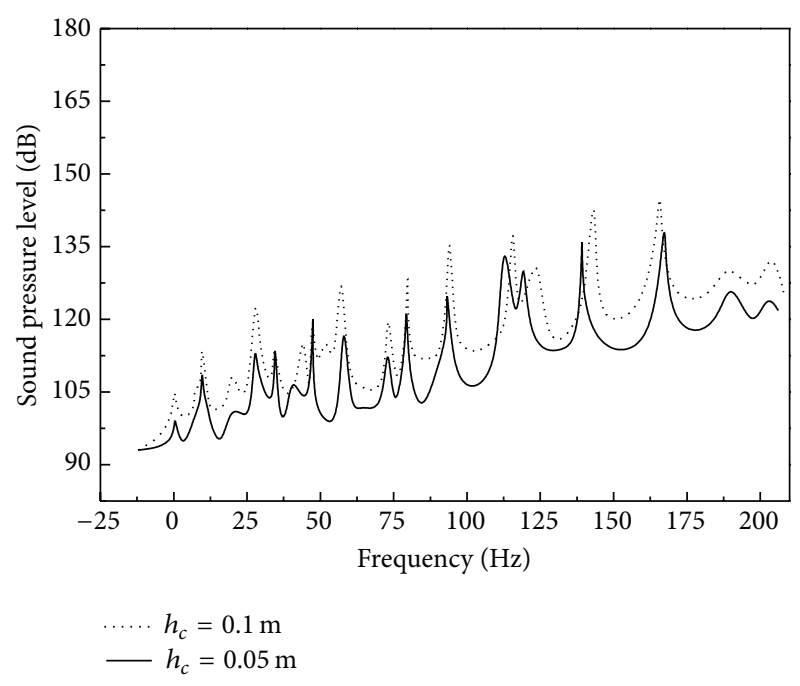

FIGURE 12: Effect of thickness of viscoelastic coating on acoustic property of the underwater structure.

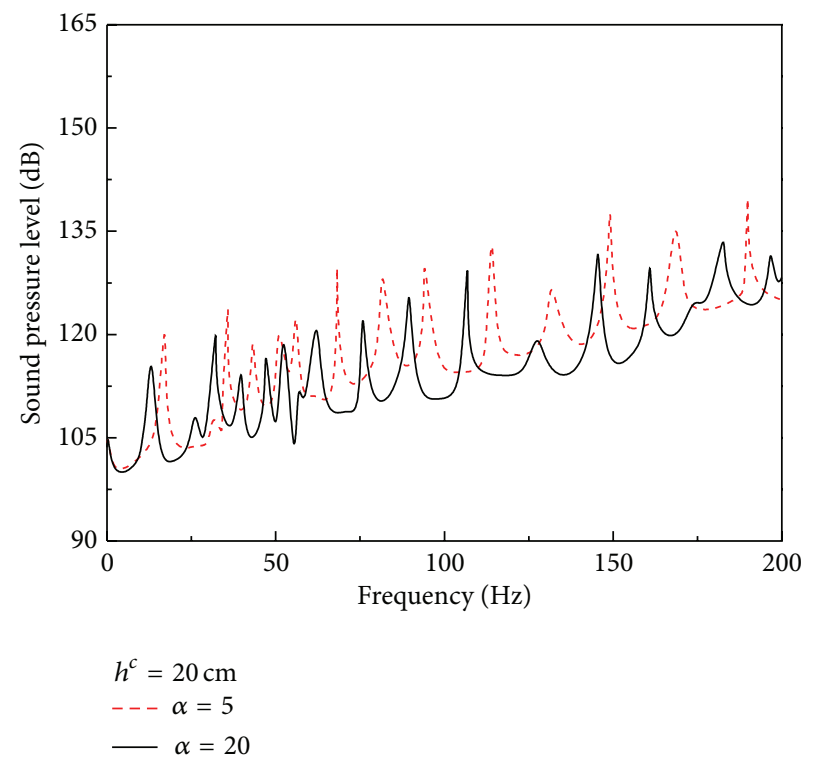

FIGURE 13: Effect of material property of viscoelastic coating on acoustic property of the underwater structure.

shown in (56). In the present analysis, the surfaces of the cylindrical and conical shell are divided into the six-point quadrilateral elements. The linear interpolation along the axial direction and quadratic interpolation along circumferential direction are adopted for the boundary elements. In the following, the acoustic property of the underwater structure radiated by the structural vibration is investigated and the parameters effects are discussed.

5.2. Numerical Examples on Acoustic Response of the Underwater Structure. Some numerical examples are given for the radiating acoustic response for the underwater structure with the geometrical and material parameters presented in 


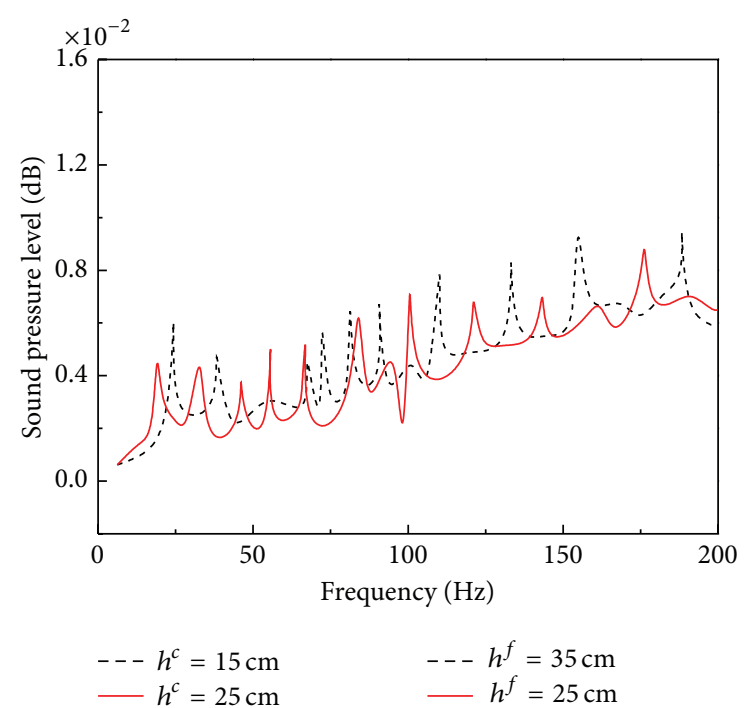

FIGURE 14: Acoustic pressure of the underwater structure with different thickness of FGM core and viscoelastic coating.

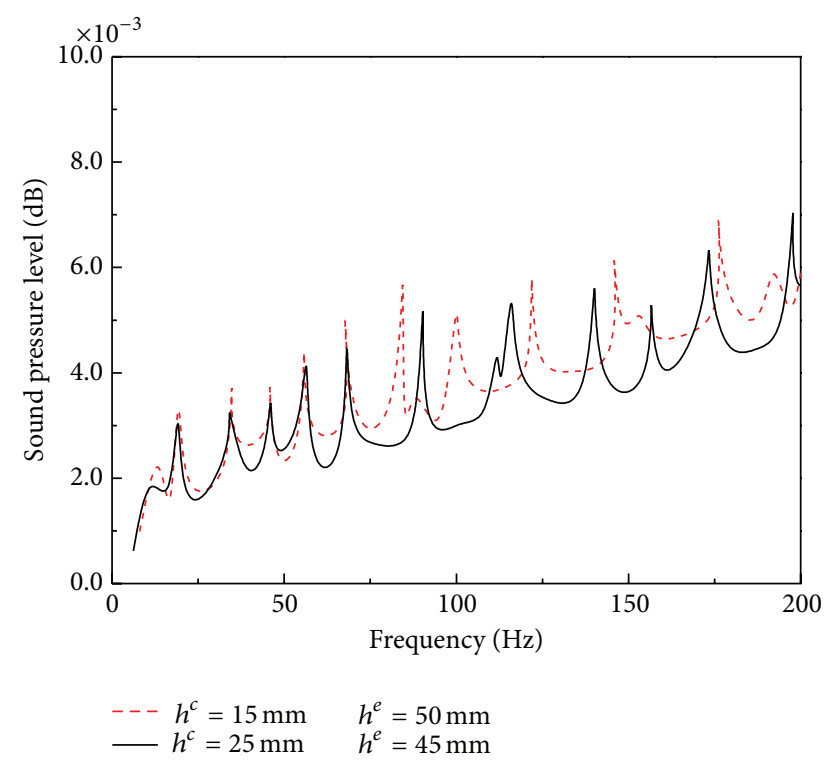

Figure 15: Acoustic pressure of the underwater structure with different thickness of FGM core and viscoelastic coating.

Tables 2 and 3. A full comprehension of acoustic property is necessary for the acoustic control analysis; the emphasis of parameters effect analysis of the underwater structure is put on the viscoelastic coating and elastic substrate in this paper.

Effect of coating thickness on radiating far-field acoustic pressure is investigated firstly. Figure 12 shows the relations of acoustic frequency and acoustic amplitude with different coating thicknesses. From the figure, we can see that a thicker coating induces a lower acoustic response. This can be explained by that a strengthened structural stiffness is obtained with the increasing of coating thickness and consequently a comparatively small vibration takes place. Another factor is the viscoelastic property, which presents

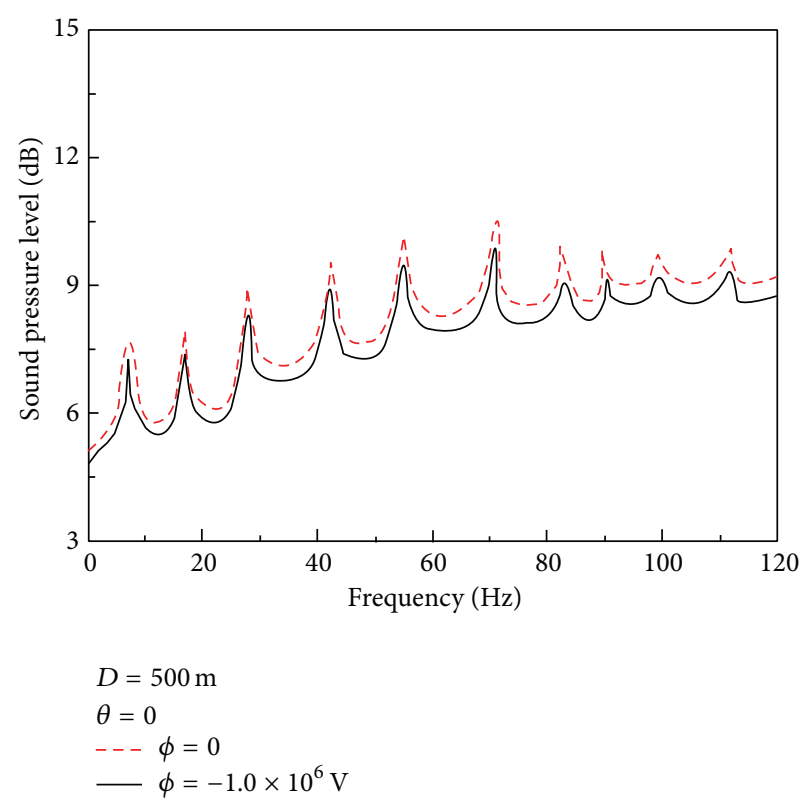

FIGURE 16: Acoustic pressure of the underwater structure when a negative electric potential is applied.

an obvious effect on the structural vibration in the form of damping effect as observed from Figure 13.

As the discussions above about effect of thickness on acoustic response, a thicker coating attenuates the acoustic pressure; however, this is a paradox for the energy consumption. Therefore, a balance between the energy consumption and the strength requirement of the underwater structure should be found. In the following, the acoustic response of the underwater structure with different thicknesses for the coating, the FGM core, and the elastic substrate are discussed when a constant total thickness of the underwater structure is set.

It can be seen from Figure 14 that an apparent variation of the acoustic response can be observed when different thicknesses for coating and FGM core are set. The change of the structure's strength is caused by the change of thickness, the fundamental vibrational frequency is also changed, and consequently the variation of the radiated acoustic frequency and amplitude can be also observed. The same conclusion can be drawn from Figure 15 in which different thickness of substrate and FGM core are set.

The piezoelectric effect on acoustic response is investigated and discussed when the geometrical and material parameters are taken the same as those in the previous example. Different electric potential are applied and the corresponding acoustic property are plot in Figures 16 and 17. It can be seen from the figure that when a positive electric potential is applied the acoustic pressure is a little greater than that when no electric potential is applied, while a negative electric potential causes a greater pressure. This reason is a negative electric potential caused a stiffener structure and consequently attenuates structural vibration, and a restrain effect on radiated acoustic pressure can be observed. 


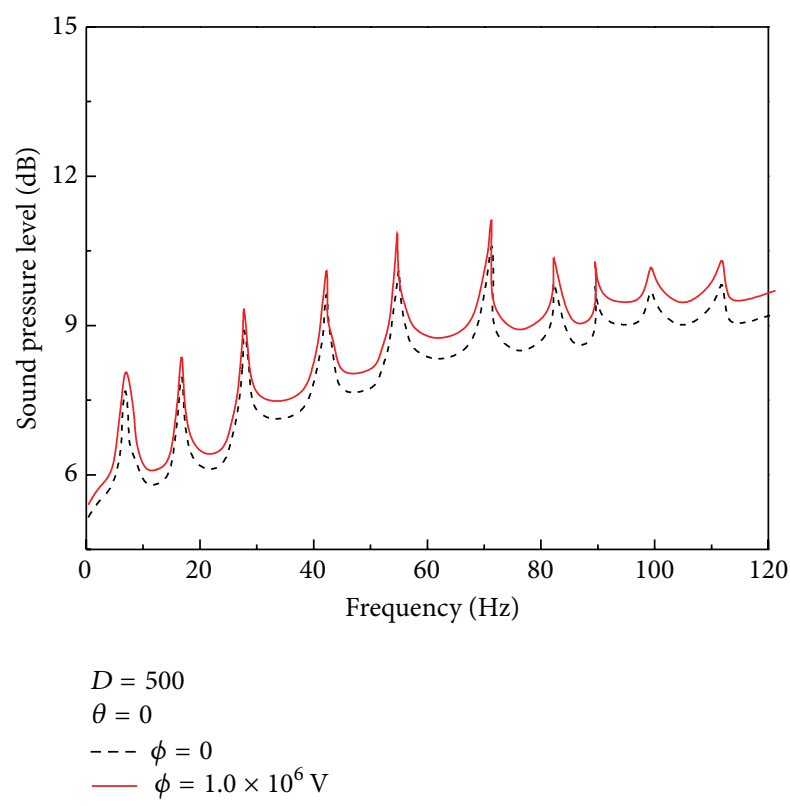

FIGURE 17: Acoustic pressure of the underwater structure when a positive electric potential is applied.

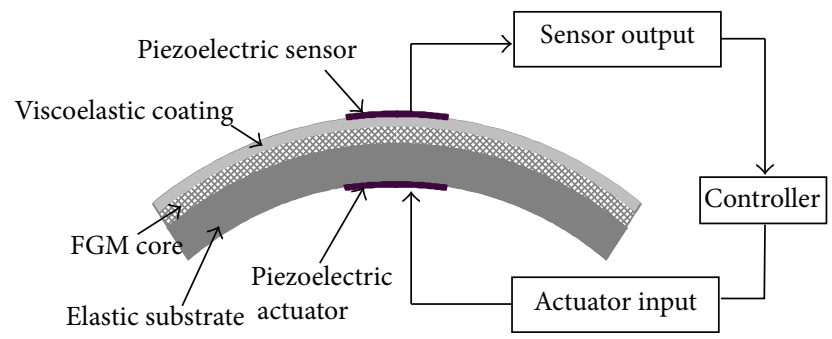

FIGURE 18: Schematic diagram of the piezoelectric control loop.

\section{Control Analysis on Vibration and Acoustic Properties of the Underwater Structure}

6.1. The Electric Potential Generated by the Active Control Loop. In this part, the adaptive control with use of piezoelectric films on the underwater structure's vibration and radiating acoustic properties are investigated.

The negative velocity feedback control algorithm coupling the direct and converse piezoelectric effects is used to realize the active control through a closed control loop (as shown in Figure 18). The piezoelectric constitutive relation is referred to (12) and the piezoelectric equilibrium equation of the piezoelectric structure is referred to (42). To achieve the integrity of the deducing of the control equations, the motion equations of the underwater structure with piezoelectric films amounting on top and bottom surfaces are written in the following:

$$
\begin{gathered}
{[M]\{\ddot{X}\}+[K]_{(e)}\{X\}+[K]_{(e \phi)} \Phi+[K]^{(v)} \otimes X_{m n}=\{F\}} \\
{[K]_{(e \phi)}\{X\}+[K]_{(\phi \phi)} \Phi=\{Q\} .}
\end{gathered}
$$

When considering the direct and inverse piezoelectric effect, the $\Phi$ generated by the mechanical effect can be given as follows according to (59):

$$
\begin{aligned}
\Phi & =\left(\{Q\}-[K]_{(e \phi)}^{T}\{X\}\right)\left(-[K]_{(\phi \phi)}^{T}\right)^{-1} \\
& =-\left([K]_{(\phi \phi)}\right)^{-1}\{Q\}+\left([K]_{(\phi \phi)}\right)^{-1}[K]_{(e \phi)}^{T}\{X\}
\end{aligned}
$$

where $\{Q\}$ is the input charge for the actuator film, and it is zero for the sensor film.

Then, the following motion equation can be obtained:

$$
\begin{aligned}
& {[M]\{\ddot{X}\}+[K]_{(e)}\{X\}+[K]_{(e \phi)}} \\
& \quad \times\left(-[K]_{(\phi \phi)}^{-1} Q+[K]_{(\phi \phi)}^{-1}[K]_{(e \phi)}^{T}\right)\{X\} \\
& \quad+[K]^{(\nu)} \otimes X_{m n}=\{F\} .
\end{aligned}
$$

The following simplified denotation is obtained as

$$
[M]\{\ddot{X}\}+[K]_{(e)}^{*}\{X\}+[K]^{(v)} \otimes X_{m n}=\{F\}-\{F\}_{(c)},
$$

where

$$
\begin{aligned}
& {[K]_{(e)}^{*}=[K]_{(e)}-[K]_{(e \phi)}[K]_{(\phi \phi)}^{-1}[K]_{(e \phi)}^{T}} \\
& \{F\}_{(c)}=[K]_{(e \phi)}[K]_{(\phi \phi)}^{-1}\left[K_{(\phi \phi)}\right]^{-1}\{Q\} .
\end{aligned}
$$

The charge output of the sensor, with poling in the $z$ direction, can be expressed in terms of spatial integration of the electric displacement over its surface as follows:

$$
Q=\frac{1}{2}\left(\int_{S\left(z=h_{b}^{(p)}\right)} D_{z} d s+\int_{S\left(z=h_{t}^{(p)}\right)} D_{z} d s\right) .
$$

Then the current in the control loop by the charge generated by the sensor can be given as

$$
I=\frac{d Q}{d t}
$$

The actuator voltage, to be applied on the bottom surface of the actuator, can be obtained when the current is converted by the control loop into the open circuit sensor voltage and then amplified with a charge of polarity as follows:

$$
\phi=-G_{i} G_{c} \frac{d Q}{d t}
$$

where $G_{i}$ is the constant gain of the amplifier and $G_{c}$ is the gain of the amplifier to provide feedback control. Assuming the top surface of the actuator is short connected with substrate and the distribution of the electric potential is linear along the thickness in the actuator, the electric field in the actuator can be written as

$$
E_{z}=-2 \frac{G_{i} G_{c}}{h^{(p)}} \frac{d Q}{d t} .
$$




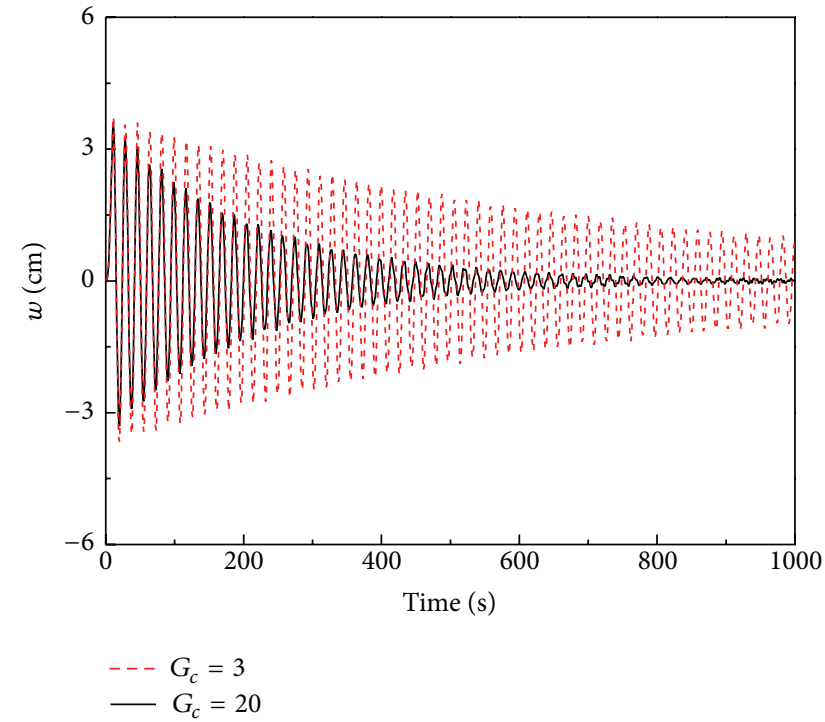

FIGURE 19: Acoustic pressure of the underwater structure when a positive electric potential is applied.

According to the constitutive relations in (12), and considering the displacement-strain relations, the electric displacement can be given in the following form:

$$
\{D\}=[e][B]\{X\}-[K]^{\phi \phi}\{E\} .
$$

When substituting the above equation to (64), the following relation can be obtained:

$$
\phi^{(s)}=-[k]^{s \phi}\{\dot{X}\},
$$

where $[k]^{s \phi}=-G_{i} G_{c} \int_{s}[e][B] d s$, and the external piezoelectric filed has been neglected.

Then the (62) of overall system equations can be written when considering the active control electric potential as follows:

$$
\begin{aligned}
{[M] } & \{\ddot{X}\}+[K]_{(e f)}[K]_{(s f)}\{\dot{X}\}+[K]_{(e)}^{*}\{X\}+[K]^{(v)} \otimes X_{m n} \\
& =\{F\}-\{F\}_{(c)} .
\end{aligned}
$$

6.2. Numerical Examples for the Vibration and Acoustic Control of the Underwater Structure. With the negative velocity feedback control algorithm, a control loop has been built for the vibration control of the underwater structure in previous section. From (70), it can be found that the sensed electric potential circulated to the actuator film is proportional to the vibration velocity of the underwater structure, which is the so-called negative velocity feedback control mechanism. It is worth noting that four pairs (sensor actuator) piezoelectric films are considered. And the sensed potentials from the sensors are different from each other. Actually, with the symmetrical loads applied, the displacement mode shows the

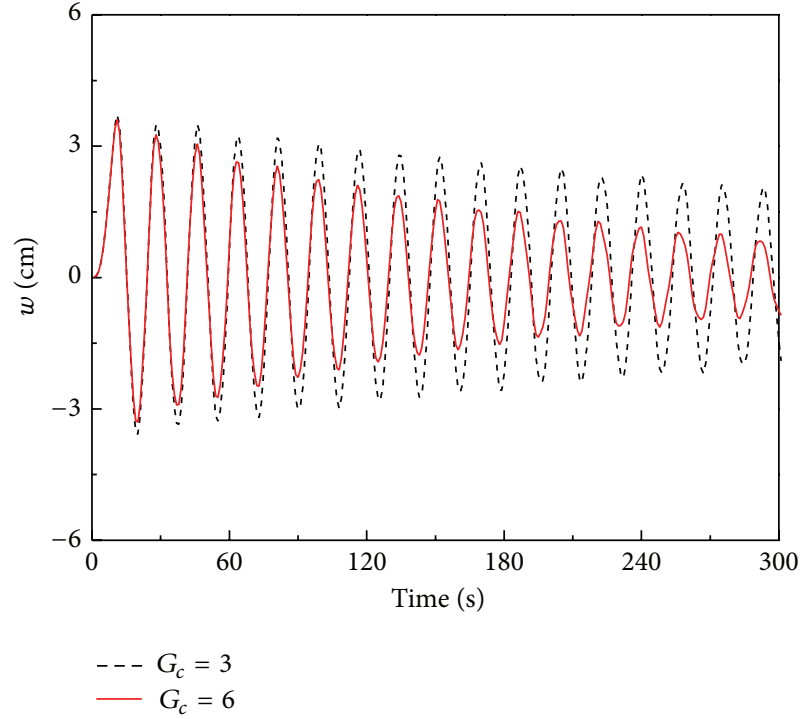

FIGURE 20: Vibration of the underwater structure with feedback control loop under active control.

symmetrical state, and the sensed potentials consequently are the same to each other. It is a different case for an unsymmetrical external loading form. In the present analysis of controlling vibration and acoustic properties of the underwater structure, the symmetrical loading forms are adopted.

Firstly, the adaptive control of the underwater structural vibration is investigated. An impact pressure is applied and the structure performs free vibration when no damping effect is considered. Though the viscoelastic property of the coating causes a vibration suppression of the underwater structure, the suppression effect is not so apparent and a comparatively longer time is needed for a complete attenuation on the underwater structure vibration. Figure 19 gives the control effect of the negative feedback control on the free vibration of the structure. It can be seen from the figure that an effective control on structural vibration of the structure can be obtained and as the control gain $G_{c}$ increases. It also can be found from the figure that the time needed to completely attenuate the structural vibration is shortened as the control gain $G_{c}$ increases, which is a useful benchmark for engineering practice.

Figures 20 and 21 present, respectively, the sensed potential and structural vibration when different control gain $G_{c}$ is set. It can be found that the sensed potential has a quarter of cycle difference with the displacement vibration, which also can be explained by (70). The sensed potential is proportional to the vibration amplitude, which proves that this control algorithm has a sensitive response to the vibration state, and consequently attenuates the structural vibration effectively.

The correspondingly acoustic pressure level is attenuated as an effective control on vibration has been obtained. An effective control for acoustic response of the underwater structure can be seen from Figures 22 and 23. With use of adaptive control method, an obvious control effect has been obtained on the acoustic pressure. By using negative feedback 


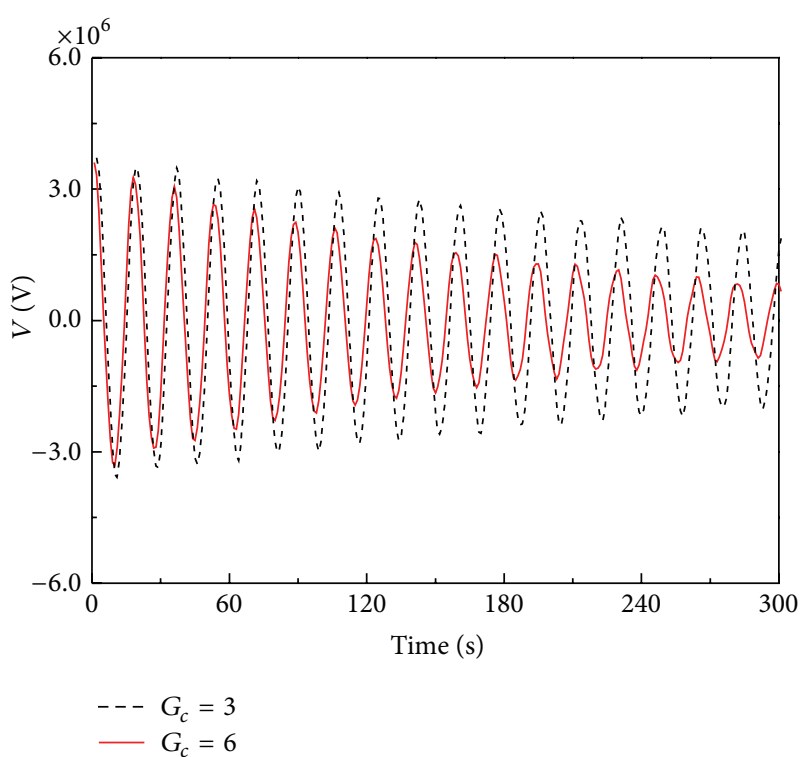

FIGURE 21: Sensed electric potential with feedback control loop under active control.

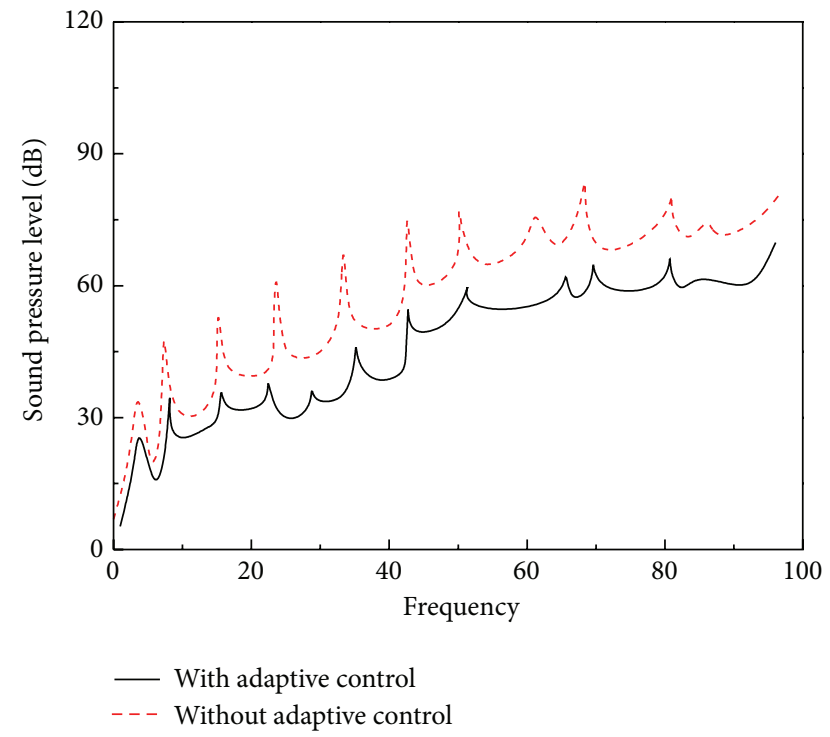

FIGURE 22: Acoustic response of underwater structure under adaptive control.

control algorithm, the vibration and acoustic control of the underwater structure obtains a feasible result which is feasible in the practical engineering.

As done above, the same piezoelectric control effect can be gotten when applying an external electric potential. But the adaptive control algorithm can have a more effective control results on the structural vibration and acoustic properties.

\section{Conclusions}

Mechanical and acoustic responses of an underwater structure have been investigated and discussed. Some numerical

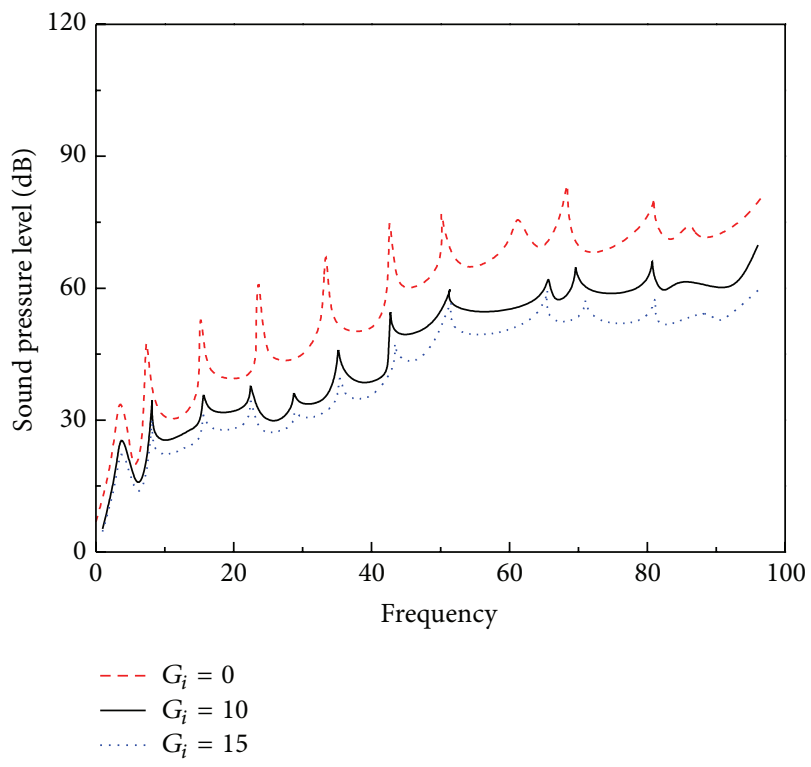

FIGURE 23: Effect of control gain on acoustic response of the underwater structure.

examples are carried out and some conclusions have been drawn.

(1) Due to the energy dissipation of the coating's viscoelastic property, the dynamic response of the underwater structure on the center of the cylindrical shell is a little small and the frequency also decreases, and it can be concluded that the vibration amplitude decreases as the time prolongs and the vibration of the structure attenuates completely with the time longer enough due to the viscoelastic energy dissipation.

(2) The electric field causes the change of the resultant force of the structure and produces effect on the underwater structure's dynamic property. The vibration amplitude increases by applying the positive electric potential and it decreases by the negative electric potential.

(3) The sensed potential is proportional to the vibration amplitude with the negative feedback control algorithm applied and has an obvious control effect on structural vibration. As an effective control on vibration has been obtained, the correspondingly acoustic pressure level has been depressed effectively.

\section{Conflict of Interests}

The authors declare that there is no conflict of interests regarding the publication of this paper.

\section{Acknowledgments}

The authors wish to thank reviewers for their valuable comments and the research is supported by the National Natural Science Foundation of China (11372105), New Century 
Excellent Talents Program in University (NCET-13-0184), Hunan Provincial Natural Science Foundation for Creative Research Groups of China (12JJ7001), and the central colleges of basic scientific research and operational costs (funded by the Hunan University).

\section{References}

[1] S. Merz, R. Kinns, and N. Kessissoglou, "Structural and acoustic responses of a submarine hull due to propeller forces," Journal of Sound and Vibration, vol. 325, no. 1-2, pp. 266-286, 2009.

[2] O. Rahmani, S. M. R. Khalili, K. Malekzadeh, and H. Hadavinia, "Free vibration analysis of sandwich structures with a flexible functionally graded syntactic core," Composite Structures, vol. 91, no. 2, pp. 229-235, 2009.

[3] A. F. Ávila, "Failure mode investigation of sandwich beams with functionally graded core," Composite Structures, vol. 81, no. 3, pp. 323-330, 2007.

[4] P. Malekzadeh, S. A. Shahpari, and H. R. Ziaee, "Threedimensional free vibration of thick functionally graded annular plates in thermal environment," Journal of Sound and Vibration, vol. 329, no. 4, pp. 425-442, 2010.

[5] T. Y. Ng, K. Y. Lam, K. M. Liew, and J. N. Reddy, "Dynamic stability analysis of functionally graded cylindrical shells under periodic axial loading," International Journal of Solids and Structures, vol. 38, no. 8, pp. 1295-1309, 2001.

[6] R. K. Bhangale and N. Ganesan, "Thermoelastic buckling and vibration behavior of a functionally graded sandwich beam with constrained viscoelastic core," Journal of Sound and Vibration, vol. 295, no. 1-2, pp. 294-316, 2006.

[7] R. Kadoli and N. Ganesan, "Buckling and free vibration analysis of functionally graded cylindrical shells subjected to a temperature-specified boundary condition," Journal of Sound and Vibration, vol. 289, no. 3, pp. 450-480, 2006.

[8] A. H. Sofiyev, "The buckling of functionally graded truncated conical shells under dynamic axial loading," Journal of Sound and Vibration, vol. 305, no. 4-5, pp. 808-826, 2007.

[9] W. Lanhe, W. Hongjun, and W. Daobin, "Dynamic stability analysis of FGM plates by the moving least squares differential quadrature method," Composite Structures, vol. 77, no. 3, pp. 383-394, 2007.

[10] S. Yin, S. Ding, A. Haghani, and H. Hao, "Data-driven monitoring for stochastic systems and its application on batch process," International Journal of Systems Science, vol. 44, no. 7, pp. 13661376, 2013.

[11] S. Yin, S. X. Ding, A. Haghan, H. Hao, and P. Zhang, "A comparison study of basic data-driven fault diagnosis and process monitoring methods on the benchmark Tennessee Eastman process," Journal of Process Control, vol. 22, pp. 15671581, 2012.

[12] M. C. Ray and R. Balaji, "Active structural-acoustic control of laminated cylindrical panels using smart damping treatment," International Journal of Mechanical Sciences, vol. 49, no. 9, pp. 1001-1017, 2007.

[13] V. Jayachandran and J. Q. Sun, "Modeling shallow-sphericalshell piezoceramic actuators as acoustic boundary control elements," Smart Materials and Structures, vol. 7, no. 1, pp. 7284, 1998.

[14] Q. Lin, Z. Liu, and Q. Wang, "Active control of structural acoustic pressure in a rectangular cavity using piezoelectric actuators," European Journal of Mechanics-A/Solids, vol. 20, no. 4, pp. 573-583, 2001.

[15] Y.-M. Fu and J.-L. Ruan, "Nonlinear active control of damaged piezoelectric smart laminated plates and damage detection," Applied Mathematics and Mechanics, vol. 29, no. 4, pp. 421-436, 2008.

[16] L. Malgaca, "Integration of active vibration control methods with finite element models of smart laminated composite structures," Composite Structures, vol. 92, no. 7, pp. 1651-1663, 2010.

[17] Y.-H. Lim, S. V. Gopinathan, V. V. Varadan, and V. K. Varadan, "Finite element simulation of smart structures using an optimal output feedback controller for vibration and noise control," Smart Materials and Structures, vol. 8, no. 3, pp. 324-337, 1999.

[18] H. Li, J. Yu, C. Hilton, and H. Liu, "Adaptive sliding-mode control for nonlinear active suspension vehicle systems using TS fuzzy approach," IEEE Transactions on Industrial Electronics, vol. 60, no. 8, pp. 3328-3338, 2013.

[19] H. Li, X. Jing, and H. R. Karimi, “Output-feedback-based $H_{\infty}$ control for vehicle suspension systems with control delay," IEEE Transactions on Industrial Electronics, vol. 61, no. 1, pp. 436-446, 2014.

[20] M. Caresta and N. J. Kessissoglou, "Acoustic signature of a submarine hull under harmonic excitation," Applied Acoustics, vol. 71, no. 1, pp. 17-31, 2010.

[21] N. D. Sharma, R. Maranganti, and P. Sharma, "On the possibility of piezoelectric nanocomposites without using piezoelectric materials," Journal of the Mechanics and Physics of Solids, vol. 55, no. 11, pp. 2328-2350, 2007.

[22] J. H. Zhang and S. R. Li, "Dynamic buckling of FGM truncated conical shells subjected to non-uniform normal impact load," Composite Structures, vol. 92, no. 12, pp. 2979-2983, 2010.

[23] A. Nosier and F. Fallah, "Non-linear analysis of functionally graded circular plates under asymmetric transverse loading," International Journal of Non-Linear Mechanics, vol. 44, no. 8, pp. 928-942, 2009. 

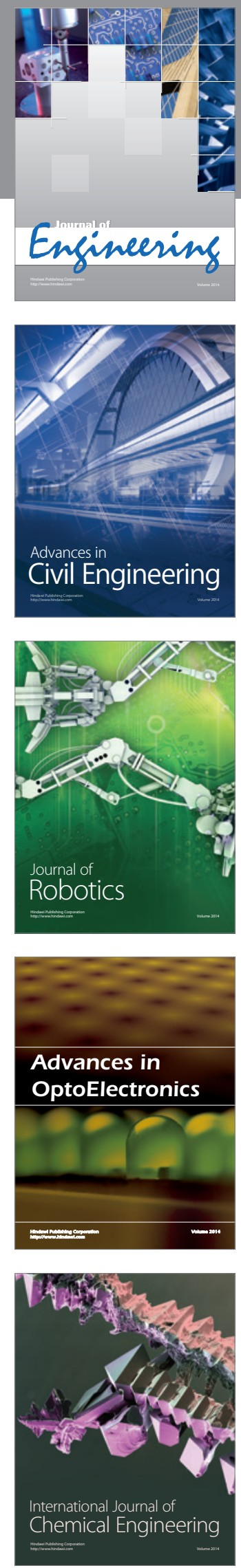

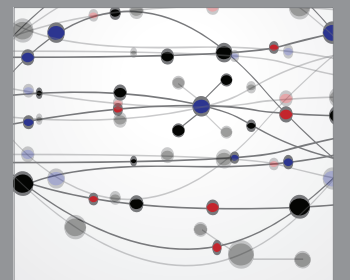

The Scientific World Journal
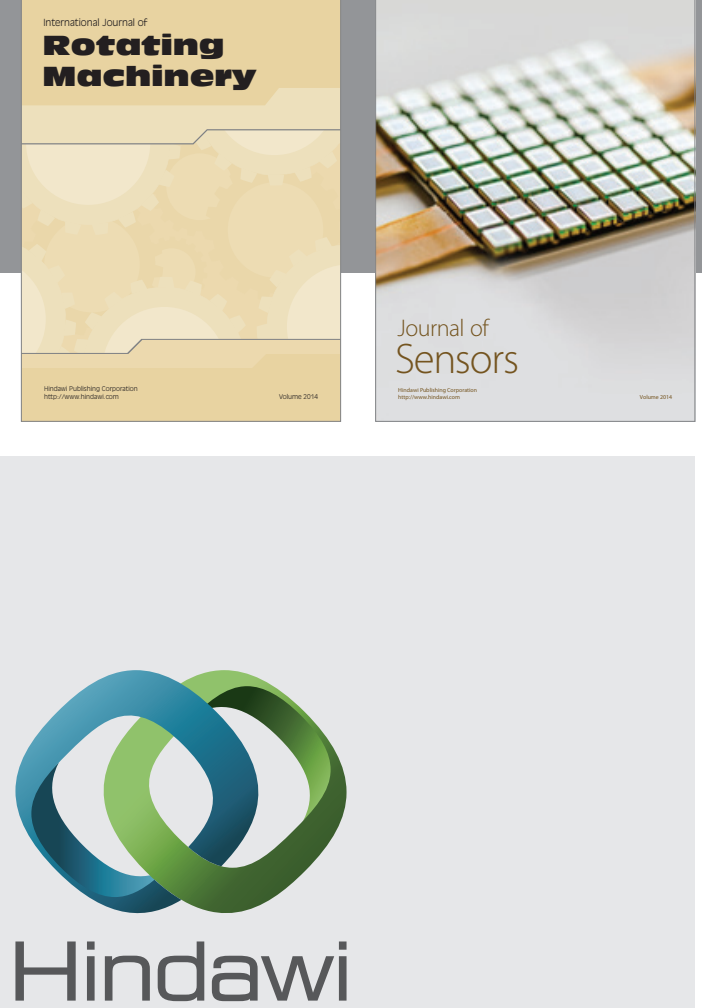

Submit your manuscripts at http://www.hindawi.com
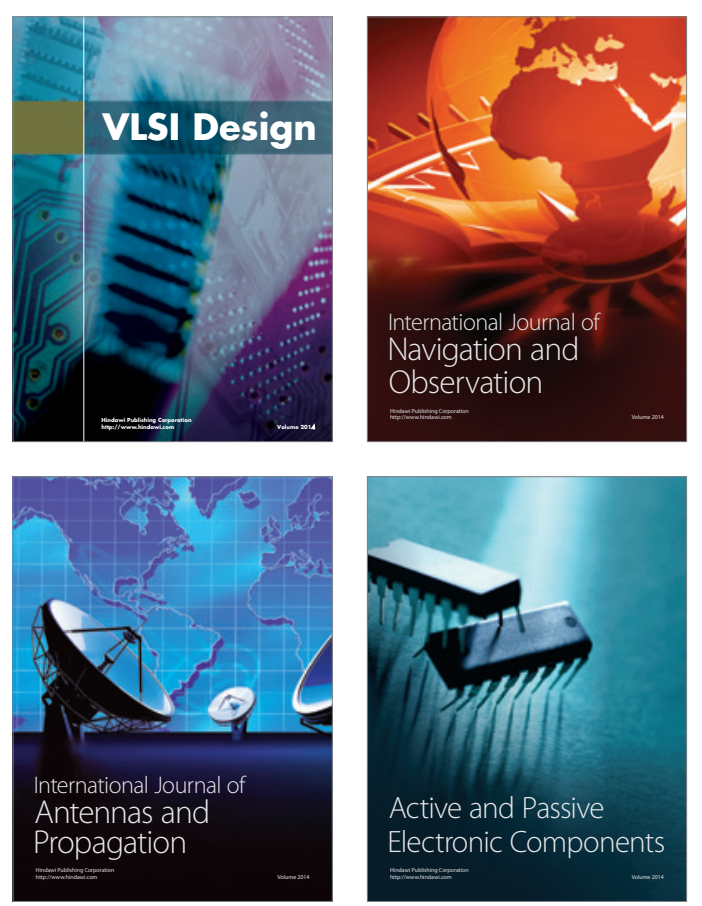
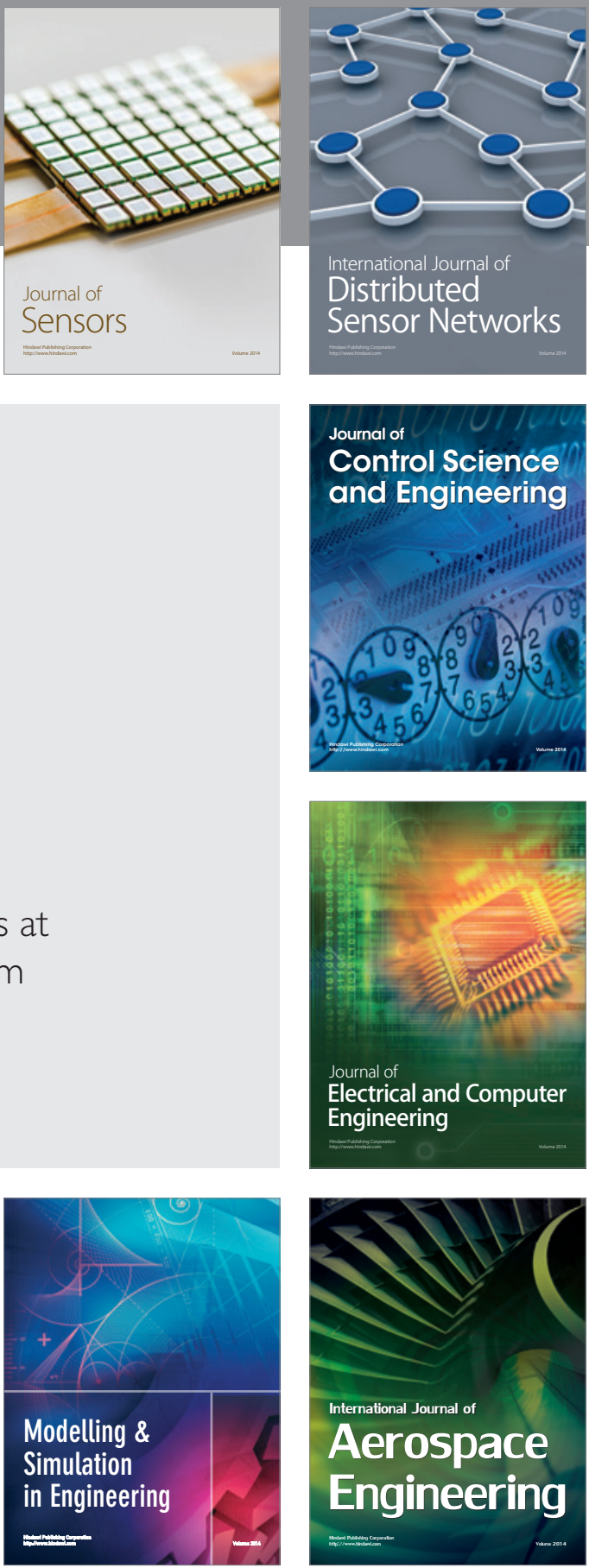

Journal of

Control Science

and Engineering
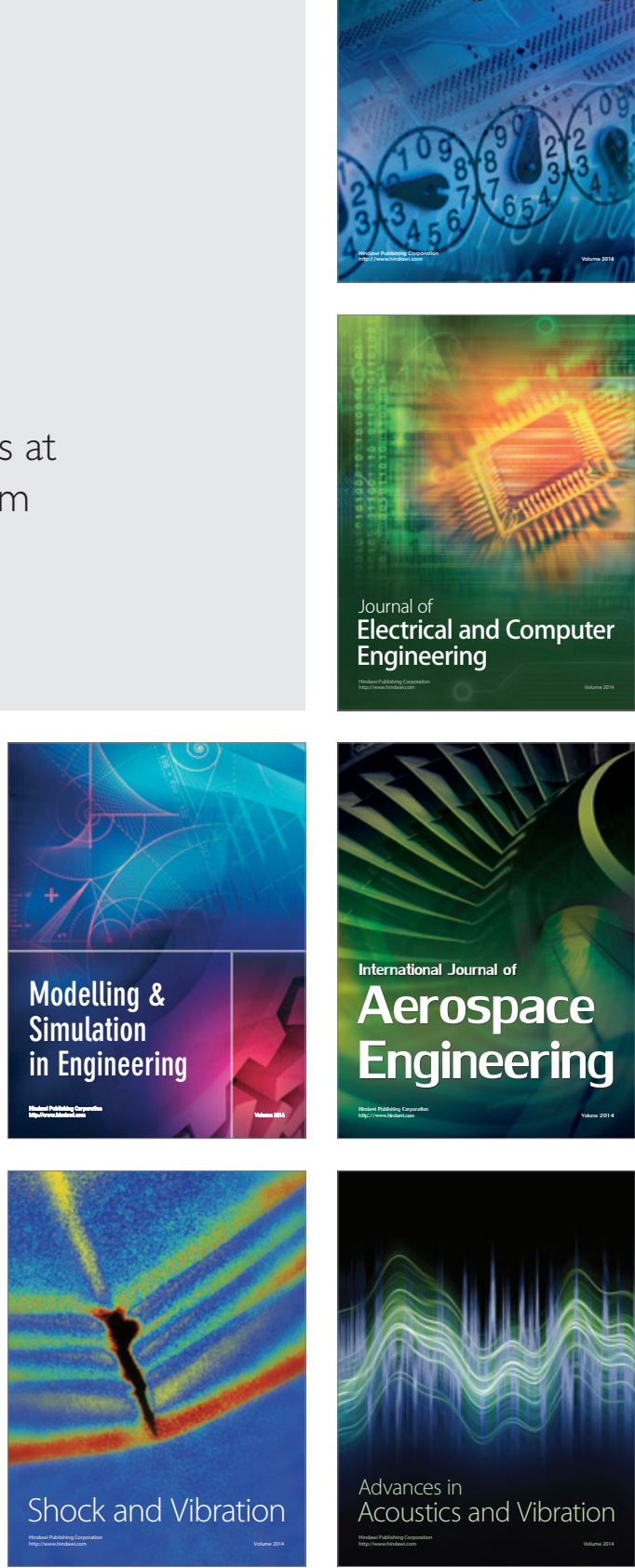